\title{
1 KCTD19 associates with ZFP541 and HDAC1 and is required 2 for meiotic exit in male mice
}

3

4 Seiya Oura ${ }^{1,2}$, Takayuki Koyano ${ }^{3}$, Chisato Kodera ${ }^{4}$, Yuki Horisawa-Takada ${ }^{4}$, Makoto 5 Matsuyama ${ }^{3}$, Kei-ichiro Ishiguro ${ }^{4}$, and Masahito Ikawa ${ }^{1,2,5}$ *

$8 \quad{ }^{1}$ Research Institute for Microbial Diseases, Osaka University, Osaka, 565-0871, Japan

$9 \quad{ }^{2}$ Graduate School of Pharmaceutical Sciences, Osaka University, Osaka, 565-0871, Japan

$10{ }^{3}$ Division of Molecular Genetics, Shigei Medical Research Institute, Okayama, 701-0202,

11 Japan

$12{ }^{4}$ Department of Chromosome Biology, Institute of Molecular Embryology and Genetics (IMEG),

13 Kumamoto University, Kumamoto, 860-0811, Japan

$14{ }^{5}$ The Institute of Medical Science, The University of Tokyo, Minato-ku, Tokyo, 108-8639, Japan

16 *Correspondence: ikawa@biken.osaka-u.ac.jp (M. I.) 


\section{Abstract}

Meiosis is a cell division process with complex chromosome events where various molecules must work in tandem. To find meiosis-related genes, we screened evolutionarily conserved and reproductive tract-enriched genes using the CRISPR/Cas9 system and identified potassium channel tetramerization domain containing 19 (Kctd19) as an essential factor for meiosis. In prophase I, Kctd19 deficiency did not affect synapsis or the DNA damage response, and chiasma structures were also observed in metaphase I spermatocytes of Kctd19 KO mice. However, spermatocytes underwent apoptotic elimination during the metaphase-anaphase transition. We were able to rescue the Kctd19 KO phenotype with an epitope-tagged Kctd19 transgene. Immunoprecipitation-mass spectrometry identified zinc finger protein 541 (ZFP541) and histone deacetylase 1 (HDAC1) as binding partners of KCTD19, indicating that KCTD19 is involved in chromatin modification. Phenotyping of Zfp541 KO spermatocytes demonstrated XY chromosome asynapsis and recurrent DNA damage in the late pachytene stage, leading to apoptosis. In summary, our study reveals that KCTD19 associates with ZFP541 and HDAC1, and that both KCTD19 and ZFP541 were essential for meiotic exit in male mice. 


\section{Author summary}

Meiosis is a fundamental process that consisting of one round of genomic DNA

replication and two rounds of chromosome segregation producing four haploid cells. To properly distribute their genetic material, cells need to undergo complex chromosome events such as a physical linkage of homologous chromosomes (termed synapsis) and meiotic recombination. The molecules involved in these events have not been fully characterized yet, especially in mammals. Using a CRISPR/Cas9-screening system, we identified the potassium channel tetramerization domain containing 19 (Kctd19) as an essential factor for meiosis in male mice. Further, we identified zinc finger protein 541 (ZFP541) and histone deacetylase 1 (HDAC1) as binding partners of KCTD19. By observing meiosis of Zfp541 knockout germ cells, we found that Zfp541 was also essential for meiotic completion. These results show that the KCTD19/ZFP541 complex plays a critical role and is indispensable for male meiosis and fertility. 


\section{Introduction}

Meiosis is a division process consisting of one round of DNA replication and two rounds of chromosome segregation, producing four haploid gametes. During meiotic prophase I, proteinaceous structures termed the synaptonemal complex (SC) are assembled on sister chromatids and form a scaffold along each homologous chromosome. The homologs begin to pair and synapse, followed by meiotic recombination yielding a physical tether between homologs (chiasmata). After completing these chromosome events, the cells transition to the first meiotic division, where homologs are segregated to the opposite poles followed by the segregation of sister chromatids in the next round of cell division. The molecules involved in these complex chromosome events are not fully characterized yet, especially in mammals, due to difficulties in culturing and genetically manipulating spermatogenic cells in vitro. Thus, knockout $(\mathrm{KO})$ of genes with testis-specific expression and evolutionarily conservation has been a powerful strategy to identify male meiosis-related genes and their functions (1). We have generated over 300 testis-enriched gene KO mice with conventional ES cell-mediated and the CRISPR/Cas9-mediated methods (2-5) and showed about one-third of them are indispensable for male fertility (6-8). During this phenotypic screening, we identified a potassium channel tetramerization domain containing 19 (Kctd19) as an evolutionarily conserved and testis expressed gene that is essential for male fertility in mice.

KCTD19 is one of 26 member KCTD family of proteins $(9,10)$ (KCTD1 -21 , KCTD12B, TNFAIP1, KCNRG, SHKBP1, and BTBD10; http://pfam.xfam.org/family/PF02214.22) which contains an N-terminal cytoplasmic tetramerisation domain (T1) usually found in voltage-gated potassium channels. The T1 domain is a subgroup of the BTB (Broad-complex, Tramtrack and Bric-à-brac) domain or 
POZ (poxvirus and zinc finger) domain family, which are often found at the $\mathrm{N}$-terminus of

$\mathrm{C} 2 \mathrm{H} 2$-type zinc-finger transcription factors. A variety of biological functions have been

identified for KCTD proteins (10), including ion channel regulation $(11,12)$, apoptosis $(13$,

14), interaction with ubiquitin ligase complexes such as cullin 3 (CUL3) $(15,16)$, and

degradation of various proteins such as histone deacetylases (HDACs) $(15,17)$. Regarding

KCTD19, Choi et al. found that ZFP541 made complex with KCTD19 and HDAC1 in male

germ cells and valproic acid (HDAC inhibitor) treatment caused hyperacetylation and

KCTD19/ZFP541 reduction in round spermatids (18), suggesting that KCTD19/ZFP541 are involved in chromatin reorganization during the post-meiotic phase (18). In this study, we generated Kctd19 KO mice using the CRISPR/Cas9 system and revealed that Kctd19 deficiency causes azoospermia due to incomplete meiosis.

82 Immunoprecipitation-mass spectrometry confirmed KCTD19, ZFP541, and HDAC1

83 interaction. Further, we also analyzed Zfp541 null spermatocyte and showed that Zfp541 is

84 necessary for pachytene exit. Our results suggested that KCTD19/ZFP541 complex functions in chromatin modification during meiosis. 


\section{$87 \quad$ Results}

\section{Kctd19 is a testis-enriched and evolutionally conserved gene}

To investigate the spatial expression of Kctd19 in mice, we performed multi-tissue RT-PCR using cDNA obtained from adult tissues and embryonic ovary, and we found that Kctd19 was specifically expressed in testis (Fig. 1A). In mice, the first wave of spermatogenesis starts soon after birth and completes within the first 35 days of postnatal development (19). To determine which stage of spermatogenic cells begin to express Kctd19, we also performed RT-PCR using cDNA obtained from postnatal testis as the first wave of spermatogenesis was progressing. The result shows that Kctd19 expression starts around postnatal day (PND) $10-12$ (Fig. 1B), which corresponds to the spermatocyte stage when the first wave of spermatogenesis reaches meiotic prophase. The PCR signals increased until PND 28 (Fig. 1B), at which time spermatid elongation starts.

The mouse KCTD19 protein comprises 950 amino-acid residues and has only one BTB domain based on SMART software (20) (Fig. 1C). Phylogenetic analysis with Clustal W2.1 (21) showed that KCTD19 was evolutionarily conserved in many mammals, including cattle, dogs, mice, and humans (Fig. 1D and S1). These results suggest that KCTD19 functions during the meiotic phase of mammalian spermatogenesis.

\section{Kctd19 is required for male fertility.}

To uncover the function of Kctd19 in vivo, we generated Kctd19 KO mice using the CRISPR/Cas9 system. To avoid affecting the proximal genes, Lrrc36 and Plekhg4, we designed the excision of the middle exons 3-12 from 16 exons total (Fig. 1E). Two crRNAs were mixed with tracrRNA and Cas9, and the prepared ribonucleoproteins (RNPs) were electroporated into murine zygotes. Of the 49 fertilized eggs that were electroporated, 40 
111 two-cell embryos were transplanted into the oviducts of three pseudopregnant female mice.

112 We obtained seven pups with the intended mutation. Subsequent mating and sequencing resulted in a heterozygous mouse with a 9620 bp deletion, referred to as Kctd19del that were genotyped with PCR (Fig. 1F and G). We confirmed Kctd19 deletion with immunoblotting (Fig. 1H) with various antibodies raised against KCTD19 protein (see Fig. 1C). The results showed complete loss of KCTD19 in Kctd19del/del testis, and the antibodies specifically recognize KCTD19 (Fig. 1H). We used rabbit polyclonal antibody (pAb) and rat monoclonal antibody (mAb) \#1 for immunoprecipitation, and rat $\mathrm{mAb} \# 2$ for immunostaining in subsequent experiments. Knockout $(\mathrm{KO})$ mice obtained by heterozygous intercrosses showed no overt gross defects in development, behavior, and survival. We caged individual Kctd19deldel male mice with wild type (wt) females for two months to analyze their fertility. Although mating plugs were often observed, Kctd19 ${ }^{\text {del/del }}$ males failed to sire any pups (Fig. 1I). We observed normal numbers of pups from Kctd19 $9^{d e l / d e l}$ females with Kctd1 $9^{\text {wt/del }}$ males $(7.8 \pm 2.2 ;$ Fig. $1 \mathrm{~J})$, indicating that Kctd19 is not required for female fertility. As Kctd19 ${ }^{\text {wtdel }}$ male mice are fully fertile, we used littermate heterozygous males as controls in some experiments. removed exon 2 (297 bp) that encodes the BTB domain by designing two crRNAs targeting intron 1 and 2 (Fig. S2A - S2C). Despite generating an inframe mutation, the deletion of the BTB domain affected Kctd19 expression or/and protein stability, and we could not detect any truncated KCTD19 protein with our antibodies (Fig. S2D and S2E). The exon 2 deleted mice showed the same phenotype as Kctd19 $9^{d e / / d e l}$ mice (Fig. S2F and $2 \mathrm{C}$ ). Therefore, we regarded

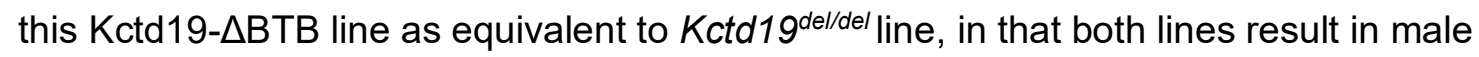

134 infertility, to corroborate that Kctd19 is essential for male fertility. 
Kctd19 del/del spermatocytes failed to complete meiosis.

When we observed testis gross morphology, Kctd19del/del testis were smaller than

those of Kctd19 $19^{w t / d e l}$ (testis/ body weight: $4.5 \pm 0.2 \times 10^{-3}[w t / d e /], 1.2 \pm 0.3 \times 10^{-3}[\mathrm{del} / \mathrm{de}]$; Fig.

$2 \mathrm{~A}$ and $2 \mathrm{~B}$ ), indicating defective spermatogenesis in $K c t d 19^{\text {del/del }}$ testis. To define the cause

testicular sections. While three germ cell layers were seen in control testis sections, only two layers of germ cells were observed in Kctd19 $9^{\text {del/del }}$ testis (Fig. 2C; low magnification). When we compared testicular cells based on the cycle of the seminiferous epithelium (22) (23), the nuclear morphology of spermatocytes was comparable between two genotypes up to seminiferous stage $\mathrm{X}-\mathrm{XI}$, corresponding to the diplotene stage (Fig. $2 \mathrm{C}$ ). In seminiferous stage XII, spermatocytes proceeded to metaphase-anaphase in Kctd19 $9^{\text {del/del }}$ testis as well as in Kctd19wt/del testis (Fig. 2C). However, the Kctd19 ${ }^{d e l / d e l}$ spermatocytes could not complete meiotic divisions and accumulated in tubules after stage XII (Fig. 2C; stage I - II). These accumulated spermatocytes underwent apoptosis (Fig. 2D) and did not develop to haploid spermatids. As a result, no mature spermatozoa were observed in the cauda epididymis (Fig. 2E). These observations suggested that $K c t d 19^{\text {del/del }}$ spermatocytes failed to complete meiosis, leading to azoospermia.

KCTD19 localized to the nuclei of prophase spermatocytes and round spermatids To determine KCTD19 localization, we performed immunostaining of testicular sections with a specific antibody against KCTD19 (Rat mAb \#2; Fig. 2F). KCTD19 signals started to appear in the nuclei of spermatocytes in seminiferous stage III - IV (Fig. 2G), corresponding to early pachytene stage. The signal continuously localized in the nuclei of 
spermatocytes (Fig. 2G; stage VII - VIII and X-XI). During the metaphase-anaphase transition in meiosis, KCTD19 signal spread throughout the cell (Fig. 2G; stage XII). The signals remained in the nuclei of round spermatids after meiotic division and disappeared in elongating spermatids. The KO phenotype and KCTD19 localization suggested that KCTD19 regulates meiosis in spermatocyte nuclei.

\section{Kctd19 $9^{\text {del/del }}$ spermatocytes showed defects in metaphase I organization.}

Due to an apparent defect in meiosis in Kctd19del/del male mice, we examined DNA double-strand breaks (DSBs) and synapsis by immunostaining $\mathrm{yH} 2 \mathrm{AX}$ and synaptonemal complex protein 3 (SYCP3), respectively. $\mathrm{yH} 2 \mathrm{AX}$ signals appeared in the leptotene/zygotene stage and disappeared in the pachytene/diplotene stage, except for the $X Y$ body (Fig. 3A and $3 \mathrm{~B})$, suggesting that $K c t d 19^{\text {del/del }}$ spermatocytes underwent DSB initiation and resolution as controls. Also, homologous chromosomes in Kctd19del/del spermatocytes synapsed in pachytene stage and desynapsed in diplotene stage remaining physically connected at chiasmata without obvious defects (Fig. 3A and 3B). However, the diplotene population declined in juvenile Kctd19del/del males (P20), but not in adult males (Fig. 3C)

To uncover the cause of apoptosis in metaphase spermatocytes, we stained spread chromosomes with Giemsa's staining. We observed a normal number of bivalent chromosomes with chiasmata (Fig. 3D), consistent with immunostaining of prophase spermatocytes. Next, we examined spindles in metaphase I spermatocytes by immunostaining of CENPC and $\alpha$-TUBULIN. Although Kctd19 ${ }^{\text {del/del }}$ spermatocytes formed spindles without apparent defects, they showed chromosome misalignment (Fig. 3E and 3FF; WT: $0 \%$, del/del: $33 \%$ ). When we stained SYCP3, we observed SYCP3 aggregates outside chromosomes, known as polycomplexes (24), more frequently in Kctd19del/del than in 
WT metaphase spermatocytes (WT: $12 \%$, del/del: $65 \%$; Fig. $3 \mathrm{G}$ and $3 \mathrm{H}$ ). These result suggested that KCTD19 is required for metaphase I organization.

\section{An epitope-tagged transgene rescues the phenotype of Kctd19del/del mice}

To exclude the possibility that the observed phenotype in Kctd19del/del males was caused by an off-target effect from CRISPR/Cas9 cleavage or an aberrant genetic modification near the Kctd19 locus, we carried out a rescue experiment by generating transgenic $(\mathrm{Tg})$ mouse lines. We mixed and injected two DNA constructs having 3xFLAGtagged Kctd19 and 3xHA-tagged Kctd19 under the testis-specific Clgn promoter (25) (Fig 4A) and established two Tg lines: one expressing only 3xHA-tagged Kctd19 (Tg line \#1) and one expressing both 3xFLAG- and 3xHA-tagged Kctd19 (Tg line \#2; Fig 4B - D). When we performed immunoprecipitation (IP) with Tg line \#2, anti-FLAG antibody-conjugated beads pull downed 3xHA-KCTD19, and vice versa (Fig. 4E and F), suggesting that KCTD19 is a homomeric protein as previously reported $(9,26)$.

When we mated Tg positive Kctd19 ${ }^{\text {del/del }}$ male mice with superovulated WT female mice (Fig. 4G), we could obtain 2-cell embryos from both Tg lines, \#1 (Fig. 4H). In Kctd19 ${ }^{\text {del/del }}$ mice carrying the 3xHA-KCTD19 transgene (\#1), the testicular size (testis/body weight: $4.7 \pm 1.6$; Fig. $4 \mathrm{~J}$ and $4 \mathrm{~K}$ ) was comparable to $\mathrm{WT}$, and spermatogenesis evaluated by HePAS staining looked normal. Further, with an anti-HA antibody, we observed a similar immunostaining pattern with rat monoclonal anit-KCT19 (Fig. 2G), indicating that the 3xHAtag did not affect KCTD19 behavior and corroborated the immunostaining results with the anti-KCTD19 antibody.

\section{KCTD19 assocaites with ZFP541 and HDAC1.}



(PND21) WT testis with non-ionic detergent (NP40) and incubated the lysate with antibodies (rabbit pAb and rat $\mathrm{mAb} \# 1$ ) and protein G-conjugate beads. The specific co-IPed proteins were visualized by SDS-PAGE and silver staining (Fig. 5A and B). When eluted samples were subjected to MS analysis, HDAC1 (histone deacetylase 1) and ZNF541 (Zinc finger protein 54; ZFP541) were reproducibly detected with both antibodies (Fig. 5C), consistent with a prior study (18). KCTD19 and HDAC1 association was confirmed by reciprocal IP with an anti-HDAC1 antibody (Fig. 5D).

HDAC1 is a modulator of chromatin structure and disruption of HDAC1 results in embryonic lethality before E10.5 (27) In previous reports, KCTDs were implicated in HDAC degradation $(15,17)$. We examine the behavior of HDAC1 in Kctd19del/del testis by immunoblotting analysis and immunostaining with the anti-HDAC1 antibody. HDAC1 protein levels and localization were comparable between $K c t d 19^{\text {del/del }}$ and WT testis (Fig. 5E and 5F). The HDAC1 staining intensity was the strongest in spermatocytes in stage $\mathrm{X}-\mathrm{XI}$ and lost in elongating spermatids (Fig. 5F), reminiscent of the KCTD19 staining pattern (Fig. 2G). These results indicated that KCTD19 works together with HDAC1 in regulating meiotic exit.

\section{Zfp541 deficient spermatocytes fail to exit the pachytene stage.}


231 ELM2 domain, and one SANT domain based on SMART software (20), indicating

KCTD19/ZFP541 binds DNA. To reveal the function of ZFP541 and its relationship with

KCTD19, we analyzed Zfp541 KO phenotype with chimeric mice (chimeric analysis) (4) (5).

To disrupt gene function completely and minimize an effect on a juxtapose gene,

Napa, we designed two sgRNAs targeting the sequence upstream of the start codon and

pairs of sgRNA/Cas9 expressing plasmids (pair 1: gRNA 1 and 3; pair 2: gRNA 2 and 4; Fig

6C). We Screened 32 clones for each pair, and obtained 13 and 11 mutant clones with

biallelic deletion for pair 1 and 2. Accounting for ES cell quality and off-target cleavages, we produced chimeric mice with one ES cell clone from pair $1(1-3 \# 2)$ and pair $2(2-4 \# 3)$

241 (Fig 6D and E).

First, we examined spermatogenesis with HePAS staining of testicular sections.

243 Almost no round spermatids with GFP were observed in chimeric mice (Fig 6F), as seen in

Kctd19del/del testis sections. Zfp541 deficient spermatocytes were eliminated by apoptosis in

stage $X-X I I$ seminiferous tubules without reaching metaphase (Fig 6G). Next, we

became weaker, although not lost, in the nuclei of $Z f p 541$ deficient spermatocytes than that intensity of HDAC1 was comparable between Zfp541 deficient and WT spermatocytes (Fig chimeric male mouse (29), in which all spermatocytes are derived from the mutant ES cells (Fig. S4A and S4B). Zfp541 deficient spermatocytes initiated DSBs in the leptotene/zygotene 
bioRxiv preprint doi: https://doi.org/10.1101/2021.02.12.430913; this version posted February 12, 2021. The copyright holder for this preprint (which was not certified by peer review) is the author/funder, who has granted bioRxiv a license to display the preprint in perpetuity. It is made available under aCC-BY 4.0 International license.

255 early pachytene spermatocytes, we could observe asynapsis of XY chromosomes (red and

256 yellow boxes in Fig. 4J). No diplotene spermatocytes were observed in the chimeric mouse,

257 consistent with histological analysis. Collectively, these results showed that Zfp541 deficient

258 spermatocytes did not reach the diplotene stage. Thus, KCTD19 may function downstream

259 of ZFP541.

260 


\section{Discussion}

In the present study, we identified Kctd19 as a male fertility-related factor by

CRISPR/Cas9-mediated screening of testis enriched genes and validated our result with

Kctd19 KO male mice (30), corroborating our results. In detailed phenotypic analyses, we found that Kctd19 KO spermatocytes failed to complete meiotic division with defects in metaphase I organization. Further, we revealed that KCTD19 associates with ZFP541 and HDAC1 by co-IP experiment using two antibodies against KCTD19. Finally, we produced chimeric mice with Zfp541-KO ES cells and showed that Zfp541 is essential for pachytene exit. eliminated by apoptosis. One of the most frequent causes of metaphase I arrest is crossover (CO) defect causing precocious homolog segregation (31, 32). However, Kctd19 KO spermatocytes had a normal number of bivalents ( 20 homologs) in metaphase I, indicating that homologs were physically connected in Kctd19 KO spermatocytes. We also observed SYCP3 polycomplexes (24), alternative SC structures, in metaphase I spermatocytes. A common cause of synaptonemal polycomplex formation is an excess amount of free SC components (24), which might be caused by premature dissociation of SC or misregulation of SC-related protein expression. However, we could not rule out the possibility that these metaphase I structural defects might be a secondary effect or phenomena in dying cells. In addition, we observed a delay of metaphase entry or elimination during prophase I in

282 juvenile Kctd19 KO males (PND20), indicating that KCTD19 may function also during prophase or that the first wave of spermatogenesis is exceptional. 
by IP-MS analysis and found ZFP541 and HDAC1 as candidate proteins, consistent with the previous report (18). Although some KCTD members have been reported to be associated with HDAC degradation, we could not observe HDAC1 reduction in KCTD19 KO testis by immunoblotting or immunostaining analysis. On the other hand, the KCTD19/ZFP541 complex is reminiscent of BTB-ZF proteins, which have another subset of the BTB domain and the Krüppel-type $\mathrm{C} 2 \mathrm{H} 2$ zinc fingers (33). Many BTB-ZF proteins have been implicated in transcriptional repressors such as N-CoR, SMRT, and HDACs via the BTB domain $(34,35)$ (36). The ELM2-SANT domain included in ZFP541 has also been shown to interact with HDAC1 $(18,37-39)$. Combined with these previous reports, our results suggested that the KCTD19/ZFP541 complex works on chromatin modification of spermatocytes with HDAC1.

We also detected CUL9 and DNTTIP1 in the IP-MS analysis with rabbit-generated antiprotects mouse eggs from aneuploidy (40) and DNTTIP1 loss causes chromosome misalignment in mitosis (41).

Finally, the chimeric analysis showed that Zfp541 KO spermatocytes failed to exit metaphase-anaphase transition. Zfp541 KO spermatocytes failed XY chromosome synapsis, and $\mathrm{yH} 2 \mathrm{AX}$ foci signals regained outside the $\mathrm{XY}$ body in the late pachytene stage, resulting in apoptosis. Again, we acknowledge that these pachytene structural defects might be secondary effects or phenomena in dying cells. 
of KCTD19 and ZFP541, which will give some insight into the molecular mechanism in male meiosis.

\section{Materials and methods}

\section{$312 \quad$ Animals}

All animal experiments were approved by the Animal Care and Use Committee of

314 the Research Institute for Microbial Diseases, Osaka University (\#Biken-AP-H30-01).

Animals were housed in a temperature-controlled environment with $12 \mathrm{~h}$ light cycles and free access to food and water. B6D2F1 (C57BL/6 × DBA2; Japan SLC, Shizuoka, Japan) mice and ICR (SLC) were used as embryo donors; B6D2F1 were used for mating and wild-type control; C57BL6/N (SLC) mice were used to collect RNA for RT-PCR and cloning. Genemanipulated mouse lines used in this study will be deposited at both the Riken BioResource through these centers.

\section{Egg collection}

To prepare eggs for knockout mouse production, female mice were superovulated by injection of CARD HyperOva (0.1 mL, Kyudo, Saga, Japan) into the abdominal cavity of B6D2F1 females, followed by injection of human chorionic gonadotropin (hCG) (7.5 units, ASKA Pharmaceutical, Tokyo, Japan). Natural mating was done with B6D2F1 males 46 48 h after CARD HyperOva injection. After 19-21 h, cumulus-intact eggs were collected and treated with $0.33 \mathrm{mg} / \mathrm{mL}$ hyaluronidase (Wako, Osaka, Japan) for $5 \mathrm{~min}$ to remove cumulus 
cells for genome editing. Obtained eggs were cultured in $\mathrm{KSOM}$ medium at $37^{\circ} \mathrm{C}$ under $5 \%$

CO2 until subsequent treatments.

\section{Generation of Kctd19 deletion and Kctd19-APOZ/TAZ mice}

Kctd19 deletion mice and Kctd19- $\triangle \mathrm{POZ} / \mathrm{TAZ}$ mice were generated by electroporation

tracrRNAs (Sigma-Aldrich, St. Louis, MO, USA) and crRNA (Sigma-Aldrich). The target of gRNA and Cas9 were as follows: for pronuclear injection, $20 \mathrm{ng} / \mu \mathrm{L} g R N A$, and $100 \mathrm{ng} / \mu \mathrm{L}$

Cas9 nucleases. The obtained complex was electroporated into fertilized eggs using a

NEPA21 electroporator (NEPA GENE, Chiba, Japan). The electroporated eggs were transplanted into the oviduct ampulla of pseudopregnant mice (ICR; 10 embryos per ampulla) on the following day. After 19 days, pups were delivered through Caesarean section and placed with foster mothers (ICR). To generate heterozygous mutant mice, F0 mice were mated with WT B6D2F1. Mouse colonies with a 9612 bp deletion and a 2172 bp deletion were maintained by sibling mating and used for the phenotype analysis of Kctd19 deletion and Kctd19- $\mathrm{POZ}$, respectively. The genotyping primers (GeneDesign, Osaka, Japan) and amplification conditions are available in Table S1.

\section{Generation of 3xFLAG-Kctd19 and Kctd19-3xHA transgenic mice}


and injected into the pronucleus of fertilized eggs. The injected eggs were transplanted into

the oviduct ampulla of pseudopregnant mice (ICR; 10 embryos per ampulla) the following day.

After 19 days, pups were delivered through Caesarean section and placed with foster mothers

(ICR). Offspring carrying both the 3xFLAG tag-Kctd19 and Kctd19-3xHA transgenes and mice

carrying only $3 \mathrm{xHA}$ tag-Kctd19 transgene were used in this study. The genotyping primers

(GeneDesign) are available in Table S1.

Generation of Zfp541 KO ES cells and chimeric mice.

described (5). Briefly, EGR-G01 ES cells were transfected with two pX459 plasmids

(Addgene plasmid \#62988) with the target sequences (Table S1), and colonies were

selected after transient puromycine selection. ES cells with normal karyotypes were injected

into ICR embryos and chimeric blastocysts were transferred into the uteri of pseudopregnant

females to produce chimeric offspring. Chimeric mals with high ES cell contribution were used for experiments.

\section{Cell Lines}

EGR-G01 ES cells were generated in the lkawa Lab (28) and cultured in KnockOut

DMEM (108297-018, Thermo Fisher Scientific) supplemented with 1\% Penicillin-

Streptomycin- Glutamine, 55 нM 2-mercaptoethanol, 1\% Non-Essential Amino Acid Solution

Scientific), $30 \mu \mathrm{M}$ Adenosine (A4036, Sigma- Aldrich, St. Louis, MO, USA), $30 \mu \mathrm{M}$ 
(U3003, Sigma-Aldrich), $10 \mu \mathrm{M}$ Thymidine (T1895, Sigma-Aldrich), $100 \mathrm{U} / \mathrm{ml}$ mouse LIF, and 20\% FCS (51650-500, Biowest, Nuaillé, France).

\section{Bacterial strains}

Escherichia coli (E. coli) strain DH5a (Toyobo, Osaka, Japan) and BL21(de3) pLysS

(C606003, ThermoFisher Scientific) were used for DNA cloning and protein expression,

respectively. E. coli cells were grown in LB or $2 \times Y T$ medium containing $100 \mathrm{mg} / \mathrm{L}$ ampicillin and were transformed or cloned using standard methods.

\section{Production of antibodies against KCTD19}

A polyclonal antibody against mouse KCTD19 was generated by immunizing rabbits with the synthetic peptide KRAITLKDWGKQRPKDRES corresponding to amino acids 747-

392 (GE healthcare), and the expression vector was transformed into E. coli strain BL21 (de3) limited dilution.

\section{Sequence comparison analysis}


RT-PCR

Using TRIzol reagent (15596-018, ThermoFisher Scientific), total RNA was isolated from multiple adult tissues of C57BL6/N mice, testes ranging from 1 to 35-day-old mice, and

embryonic ovaries of PND 11.5-19.5. cDNAs were prepared using SuperScript IV Reverse

Transcriptase (180-90050, ThermoFisher Scientific) following the manufacturer's instructions.

Polymerase chain reaction (PCR) was performed using KOD Fx neo (KFX-201, TOYOBO,

Osaka, Japan). The primers (GeneDesign) and amplification conditions for each gene are summarized in Table S1.

\section{Genotype analysis}

PCR was performed using KOD FX neo (KFX-201, TOYOBO). The primers

414 (GeneDesign) and amplification conditions for each gene are summarized in Table S1. PCR

products were purified using a Wizard SV Gel and PCR Clean-Up System (Promega, Madison,

WI, USA) kit, and Sanger sequenced was done using sequence primers listed in Table S1.

\section{Fertility analysis of KO mice}

To examine fertility, sexually mature male mice were housed with wild-type females

(B6DF1) for at least three months. Both plug and pup numbers were recorded at approximately

$10 \mathrm{AM}$ to determine the number of copulations and litter size.

Immunoblotting

Proteins from testis were extracted using NP40 lysis buffer [50mM Tris- $\mathrm{HCl}(\mathrm{pH} 7.5)$, 
reducing conditions and transferred to polyvinylidene fluoride (PVDF) membrane using the Trans Blot Turbo system (BioRad, Munich, Germany). After blocking with $10 \%$ skim milk (232100, Becton Dickinson, Cockeysville, MD, USA), the membrane was incubated with primary antibody overnight at $4^{\circ} \mathrm{C}$, and then incubated with HRP-conjugated secondary antibody for $1 \mathrm{~h}$ at room temperature. Chemiluminescence was detected by ECL Prime Western Blotting Detection Reagents (RPN2232, GE Healthcare, Chicago, IL, USA) using the Image Quant LAS 4000 mini (GE Healthcare). The antibodies used in this study are listed in Table S2.

\section{Morphological and histological analysis of testis}

To observe testis gross morphology and measure testicular weight, 11-12 week-old male mice were euthanized after measuring their body weight. The whole testis was observed using BX50 and SZX7 (Olympus, Tokyo, Japan) microscopes. For histological analysis, testes were fixed with Bouin's fixative (16045-1, Polysciences, Warrington, PA, USA) at $4^{\circ} \mathrm{C} O / \mathrm{N}$, dehydrated in increasing ethanol concentrations and $100 \%$ xylene, embedded in paraffin, and sectioned $(5 \mu \mathrm{m})$. The paraffin sections were hydrated with Xylene and decreasing ethanol concentrations and treated with $1 \%$ periodic acid (26605-32, Nacalai Tesque, Kyoto, Japan) for $10 \mathrm{~min}$, treated with Schiff's reagent (193-08445, Wako) for 20 min, counterstained with Mayer's hematoxylin solution (131-09665, Wako) for 3 min, dehydrated in increasing ethanol concentrations, and finally mounted with Permount (SP15-100-1, Ferma, Tokyo, Japan). The sections were observed using a BX53 (Olympus) microscope. 
Apoptosis Detection Kit (MK500, Takara Bio Inc., Shiga, Japan), according to the

manufacturer's instruction. Briefly, testes were fixed with Bouin's fixative, embedded in paraffin, and sectioned $(5 \mu \mathrm{m})$. After paraffin removal, the slides were boiled in citrate buffer $(\mathrm{pH} 6.0$; 1:100; ab93678, abcam, Cambridge, UK) for $10 \mathrm{~min}$ and incubated in $3 \% \mathrm{H}_{2} \mathrm{O}_{2}$ at room temperature for $5 \mathrm{~min}$ for endogenous peroxidase inactivation, followed by a labeling reaction with TdT enzyme and FITC-conjugated dUTP at $37^{\circ} \mathrm{C}$ for $1 \mathrm{~h}$. conjugated anti-FITC antibody at $37^{\circ} \mathrm{C}$ for $30 \mathrm{~min}$. The section was then incubated in ImmPACT DAB (SK-4105, Vector Laboratories, Burlingame, CA, USA) working solution, counterstained with Mayer's hematoxylin solution for $3 \mathrm{~min}$, dehydrated in increasing ethanol concentrations, and finally mounted with Permount. The sections were observed using a BX53 (Olympus) microscope.

\section{Immunostaining of testes} and sectioned $(5 \mu \mathrm{m})$. After paraffin removal, the slides were boiled in $\mathrm{pH} 6.0$ citrate buffer for $10 \mathrm{~min}$, blocked and permeabilized in $10 \%$ goat serum and $0.1 \%$ TritonX-100 for $20 \mathrm{~min}$ in PBS, and incubated with primary antibody overnight at $4^{\circ} \mathrm{C}$ or $1 \mathrm{~h}$ at room temperature in blocking solution; $1 \mathrm{~h}$ incubation was performed when using rat monoclonal anti-KCTD19 antibody. After incubation with AlexaFlour488/546-conjugated secondary antibody (1:200) at room temperature for $1 \mathrm{~h}$, samples are counterstained with Hoechst 33342 (1:2000; H3570, 
Seminiferous tubule stages were identified based on the morphological

characteristics of the germ cell nuclei and acrosome staining with AlexaFlour488/568conjugated lectin PNA (L21409/L32458, Thermo Fisher Scientific). The sections were observed using a BX53 (Olympus) microscope and a Nikon Eclipse Ti microscope connected to a Nikon C2 confocal module (Nikon, Tokyo, Japan). Fluorescent images were false-colored and cropped using ImageJ software.

Immunostaining of surface chromosome spread

Spread nuclei from spermatocytes were prepared as described (46) with slight modification. In brief, seminiferous tubules were unraveled using forceps in ice-cold DMEM

Aldrich) in DMEM $(20 \mathrm{~mL})$ at $37^{\circ} \mathrm{C}$ for $15 \mathrm{~min}$. After 3 washes with DMEM, the tubules were transferred to $20 \mathrm{~mL}$ trypsin/DNasel medium [0.025 w/v\% trypsin, $0.01 \mathrm{w} / \mathrm{v} \%$ EDTA, $10 \mathrm{U}$ DNase in DMEM] and incubated at $37^{\circ} \mathrm{C}$ for $10 \mathrm{~min}$. After adding $5 \mathrm{~mL}$ of heat-inactivated $\mathrm{FCS}$ and pipetting, the solution was filtered through $59 \mu \mathrm{m}$ mesh (N-N0270T, NBC Meshtec inc., Tokyo, Japan) to remove tubule debris. The collected testicular cells were resuspended in hypotonic solution [100 mM sucrose] and $10 \mu \mathrm{L}$ of the suspension was dropped onto a slide glass with $100 \mu \mathrm{L}$ of fixative solution [100 $\mu \mathrm{L}$ of $1 \%$ PFA, $0.1 \%(v / v)$ Triton $\mathrm{X}-100]$. The slides were then air-dried and washed with PBS containing 0.4\% Photo-Flo 200 (1464510, Kodak Alaris, NY, USA) or frozen for longer storage at $-80^{\circ} \mathrm{C}$.

The spread samples were blocked with $10 \%$ goat serum in PBS and then incubated with primary antibodies overnight at $4^{\circ} \mathrm{C}$ in blocking solution. After incubation with AlexaFlour 
observed using a BX53 (Olympus) microscope.

\section{Giemsa staining of metaphase I chromosome spread}

For preparing metaphase chromosome spreads, seminiferous tubules were

unraveled using forceps in ice-cold PBS and transferred to a $1.5-\mathrm{mL}$ tube with $1 \mathrm{~mL}$ of at room temperature. After filtration with a $59 \mu \mathrm{m}$ mesh and centrifugation, the cells were resuspended in $8 \mathrm{~mL}$ of hypotonic solution [ $1 \%$ sodium citrate] and incubated for $5 \mathrm{~min}$ at room temperature. The suspension was centrifuged and $7 \mathrm{~mL}$ of supernatant was aspirated. The cells were then resuspended in the remaining $1 \mathrm{~mL}$ of supernatant and $7 \mathrm{~mL}$ of Carnoy's washes with Carnoy's Fixative, the cells were resuspended $\sim 0.5 \mathrm{~mL}$ of Carnoy's Fixative and dropped onto a wet glass slide. The slide was stained with Giemsa Stain Solution (079-04391, wako) and observed using a BX53 (Olympus) microscope.

\section{Immunostaining of metaphase I cells}

For cytological analysis of metaphase I cells, seminiferous tubule squashes were

performed as previously described (47). In brief, seminiferous tubules were incubated in

fix/lysis solution [0.1 \% TritonX-100, $0.8 \%$ PFA in PBS] at room temperature for 5 min. Tubule bunches were then put on glass slides with $100 \mu \mathrm{L}$ of fix/lysis solution, minced into $1.0 \sim 3.0$ $\mathrm{mm}$ segments with forceps, and arranged so that no tubule segment overlaped. After removing the excess amount of fix/lysis solution, a coverslip and pressure was applied to disperse cells, and a needle. For longer storage, the slide glasses were kept at $-80^{\circ} \mathrm{C}$ with the coverslip. 
The slides were blocked and permeabilized in $10 \%$ goat serum and $0.1 \%$ Triton $\mathrm{X}$ with AlexaFlour 488/546-conjugated secondary antibody (1:200) at room temperature for $1 \mathrm{~h}$, samples are counterstained with Hoechst 33342 (1:2000) and mounted with Immu-Mount. Zstack images were taken using a BZ-X700 (kyence, Osaka, Japan) microscope and stacked using ImageJ software. The antibodies used in this study are listed in Table S2.

Immunoprecipitation and mass spectrometry analysis

Proteins from testis were extracted using NP40 lysis buffer [50 mM Tris- $\mathrm{HCl}(\mathrm{pH} 7.5)$, incubated for $1 \mathrm{~h}$ at $4^{\circ} \mathrm{C}$ and washed 3 times with NP40 lysis buffer. Co-immunoprecipitated products were then eluted with $18 \mu \mathrm{L}$ of $100 \mathrm{mM} \mathrm{Gly}-\mathrm{HCl}(\mathrm{pH} 2.5)$ and neutralized with $2 \mu \mathrm{L}$ of

$1 \mathrm{M}$ Tris. The antibodies used in this study are listed in Table S2. Half of the eluted amount was subjected to SDS-PAGE and silver staining (06865-81, Nacalai Tesque). The remaining half amount was subjected to mass spectrometry (MS) analysis. Science, Tokyo, Japan). The resultant peptides were subjected to nanocapillary reversedphase LC-MS/MS analysis using a C18 column $(25 \mathrm{~cm} \times 75 \mathrm{um}, 1.6 \mu \mathrm{m}$; lonOpticks, Victoria, Australia) on a nanoLC system (Bruker Daltoniks, Bremen, Germany) connected to a tims

543 TOF Pro mass spectrometer (Bruker Daltoniks) and a modified nano-electrospray ion source

544 (CaptiveSpray; Bruker Daltoniks). The mobile phase consisted of water containing $0.1 \%$ formic acid (solvent $\mathrm{A}$ ) and acetonitrile containing $0.1 \%$ formic acid (solvent $\mathrm{B}$ ). Linear gradient elution 
was carried out from $2 \%$ to $35 \%$ solvent $B$ for $18 \mathrm{~min}$ at a flow rate of $400 \mathrm{~nL} / \mathrm{min}$. The ion spray voltage was set at $1.6 \mathrm{kV}$ in the positive ion mode. lons were collected in the trapped ion mobility spectrometry (TIMS) device over $100 \mathrm{~ms}$ and MS and MS/MS data were acquired over an $\mathrm{m} / \mathrm{z}$ range of $100-1,700$. During the collection of MS/MS data, the TIMS cycle was adjusted to $1.1 \mathrm{~s}$ and included $1 \mathrm{MS}$ plus 10 parallel accumulation serial fragmentation (PASEF)-MS/MS scans, each containing on average $12 \mathrm{MS} / \mathrm{MS}$ spectra (>100 Hz), and nitrogen gas was used as the collision gas. against the SwissProt database. Quantitative value and fold exchange were calculated by Scaffold4 (Proteome Software, Portland, OR, USA) for MS/MS-based proteomic studies.

\section{Chimeric analysis} or immunohistochemistry. The antibodies used in this study are listed in Table S2.

\section{Author contributions}

S.O. and M.I. conceived and designed the research; S.O. performed experiments; paper.

\section{Declaration of interests}


bioRxiv preprint doi: https://doi.org/10.1101/2021.02.12.430913; this version posted February 12, 2021. The copyright holder for this preprint (which was not certified by peer review) is the author/funder, who has granted bioRxiv a license to display the preprint in perpetuity. It is made available under aCC-BY 4.0 International license.

\section{Data availability statement}

The authors declare that the data that support the findings of this study are available from the corresponding author upon request. 


\section{Acknowledgement}

We would like to thank Eri Hosoyamada and Mei Koyama for their technical

576 assistance and Dr. Julio M. Castaneda for the critical reading of the manuscript. This work was

577 supported by the Ministry of Education, Culture, Sports, Science and Technology

578 (MEXT)/Japan Society for the Promotion of Science (JSPS) KAKENHI grants (JP19J21619 to

579 S.O., JP19H05743 to K.I., and JP19H05750 to M.I.); Japan Agency for Medical Research and

580 Development (AMED) grant JP18gm5010001 to M.I.; Takeda Science Foundation grants to

581 M.I. The funders had no role in the study design, data collection and analysis, decision to

582 publish, or preparation of the manuscript.

583 


\section{References}

1. Matzuk MM, Lamb DJ. The biology of infertility: research advances and clinical challenges. Nat Med. 2008;14(11):1197-213. mice by pronuclear injection of circular plasmid expressing Cas9 and single guided RNA. Sci Rep. 2013;3:3355. analysis with newly established EGFP/DsRed2-tagged ES cells identify HYDIN as essential for spermiogenesis in mice. Exp Anim. 2019;68(1):25-34. interaction with the oocyte. J Clin Invest. 2010;120(4):984-94.

603 8. Miyata H, Castaneda JM, Fujihara Y, Yu Z, Archambeault DR, Isotani A, et al. Genome 604 engineering uncovers 54 evolutionarily conserved and testis-enriched genes that are not required 605 for male fertility in mice. Proc Natl Acad Sci U S A. 2016;113(28):7704-10.

606 9. Liu Z, Xiang Y, Sun G. The KCTD family of proteins: structure, function, disease relevance.

607 Cell Biosci. 2013;3(1):45. 
609 family involved in neurodevelopmental and neuropsychiatric disorders. CNS Neurosci Ther.

$610 \quad 2019 ; 25(7): 887-902$.

611 11. Azizieh R, Orduz D, Van Bogaert P, Bouschet T, Rodriguez W, Schiffmann SN, et al.

612 Progressive myoclonic epilepsy-associated gene KCTD7 is a regulator of potassium conductance in

613 neurons. Mol Neurobiol. 2011;44(1):111-21.

614 12. Usman H, Mathew MK. Potassium channel regulator KCNRG regulates surface expression of Shaker-type potassium channels. Biochem Biophys Res Commun. 2010;391(3):1301-

5.

617 13. Kim DM, Chung KS, Choi SJ, Jung YJ, Park SK, Han GH, et al. RhoB induces apoptosis

618 via direct interaction with TNFAIP1 in HeLa cells. Int J Cancer. 2009;125(11):2520-7.

619 14. Nawa M, Kage-Nakadai E, Aiso S, Okamoto K, Mitani S, Matsuoka M. Reduced expression of BTBD10, an Akt activator, leads to motor neuron death. Cell Death Differ. 2012;19(8):1398-407.

Identification and characterization of KCASH2 and KCASH3, 2 novel Cullin3 adaptors suppressing histone deacetylase and Hedgehog activity in medulloblastoma. Neoplasia. $2011 ; 13(4): 374-85$. Recognition Is Not a Universal Property among KCTD Proteins. PLoS One. 2015;10(5):e0126808. deacetylase and Cullin3-REN(KCTD11) ubiquitin ligase interplay regulates Hedgehog signalling through Gli acetylation. Nat Cell Biol. 2010;12(2):132-42.

631 18. Choi E, Han C, Park I, Lee B, Jin S, Choi H, et al. A novel germ cell-specific protein, 
SHIP1, forms a complex with chromatin remodeling activity during spermatogenesis. J Biol Chem.

19. Kluin PM, Kramer MF, de Rooij DG. Spermatogenesis in the immature mouse proceeds acid-STRA8 signaling intersects with periodic germ-cell competencies to regulate spermatogenesis. cycle of the seminiferous epithelium and germ cell renewal. Am J Anat. 1956;99(3):391-413. Good Thing? Trends Genet. 2020;36(11):833-44. of the testis-specific gene 'calmegin' promoter sequence and its activity defined by transgenic mouse experiments. FEBS Lett. 1995;368(3):509-12. domain of the Shaker potassium channel. Nature. 1998;392(6679):945-8. 
germline transmission-failed knockout chimeras. Transgenic Res. 2013;22(1):195-200. imprinting of $\mathrm{XX}$ spermatogonia and $\mathrm{XX}$ oocytes recovered from $\mathrm{XX}<-->\mathrm{XY}$ chimeric testes. Proc Natl Acad Sci U S A. 2005;102(11):4039-44. meiosis-essential genes based on spermatogenesis proteome dynamics. Mol Cell Proteomics. 2020. death in MLH1-deficient mouse spermatocytes: insights into regulation of spermatogenic progress. Dev Biol. 2002;249(1):85-95. arrest. Nature. 1993;362(6418):364-6. analysis of BTB domain proteins. Genome Biol. 2005;6(10):R82.

34. Huynh KD, Bardwell VJ. The BCL-6 POZ domain and other POZ domains interact with the co-repressors N-CoR and SMRT. Oncogene. 1998;17(19):2473-84. binding and transcriptional regulation by the promyelocytic leukemia zinc finger protein. J Biol Chem. 1997;272(36):22447-55. residues within the BTB domain of PLZF and Bcl-6 modulate interaction with corepressors. Mol Cell Biol. 2002;22(6):1804-18. 
functional characterization of a cell cycle associated HDAC1/2 complex reveals the structural basis structure. Nat Commun. 2020;11(1):3252. abundantly expressed in the epididymis are not essential for male fecundity in mice. Andrology. 2019;7(5):644-53. for flagellar axoneme maintenance and male mouse fertility. PLoS Genet. 2020;16(8):e1008954. antibody-producing hybridomas by using rat medial iliac lymph node cells. Cell Struct Funct. $1995 ; 20(2): 151^{-6}$

45. Sado Y, Inoue S, Tomono Y, Omori H. Lymphocytes from enlarged iliac lymph nodes as fusion partners for the production of monoclonal antibodies after a single tail base immunization attempt. Acta Histochem Cytochem. 2006;39(3):89-94. 
bioRxiv preprint doi: https://doi.org/10.1101/2021.02.12.430913; this version posted February 12, 2021. The copyright holder for this preprint (which was not certified by peer review) is the author/funder, who has granted bioRxiv a license to display the preprint in perpetuity. It is made available under aCC-BY 4.0 International license.

704 Like 5, is Required for Spermatogenesis in Micedagger. Biol Reprod. 2020;102(4):975-83.

705 47. Wellard SR, Hopkins J, Jordan PW. A Seminiferous Tubule Squash Technique for the

706 Cytological Analysis of Spermatogenesis Using the Mouse Model. J Vis Exp. 2018(132).

707

708 


\section{Figure legends}

Fig 1. Production of Kctd19 ${ }^{\text {del/del }}$ mice and fertility analysis.

711

(A) RT-PCR using multi-tissue cDNA. Actb was used as a loading control. (B) RT-PCR using postnatal testis cDNA. Actb was used as a loading control. (C) Schematic of KCTD19 protein structure and antigen position. (D) Phylogenetic tree constructed by ClustalW with KCTD19 sequences of various mammals. (E) Gene map of Kctd19. Black and white boxes indicate coding and non-coding regions, respectively. Black arrows and arrowheads indicate primers for genotyping and gRNAs for genome editing, respectively. (F) An example of genotyping PCR with two primer sets shown in E. (G) DNA sequencing for deletion verification. $(H)$ Immunoblotting with antibodies against mouse KCTD19. Red arrows indicate the expected molecular size of KCTD19. GAPDH was used as a loading control. (I) The result of mating tests. Pups/plug: 8.8 $\pm 2.4[\mathrm{WT}] ; 0[\mathrm{del} / \mathrm{de}] \mathrm{J}$ (J) Pup numbers obtained from mating pairs of Kctd19 $9^{\text {del/del }}$ females and Kctd19wt/del males $(7.8 \pm 2.2)$.

\section{Fig 2. Histological analysis of Kctd19del/del mice.}

(A) Testis morphology and (B) testis/body weight of $K c t d 19^{w t / d e l}$ and $K c t d 19^{\text {del/del }}$ adult mice at 12 weeks. Testis/body weight: $4.5 \pm 0.2 \times 10^{-3}[\mathrm{wt} / \mathrm{de} /], 1.2 \pm 0.3 \times 10^{-3}$ [del/de/]. Error bars indicate one standard deviation. (C) PAS staining of seminiferous tubules of adult mice. The seminiferous epithelium cycle was determined by germ cell position and nuclear morphology. (D) TUNEL staining of seminiferous tubules of adult mice counterstained with hematoxylin. (E) PAS staining of cauda epididymis of adult mice. (F \& G) Immunostaining of seminiferous tubules of adult mice. The seminiferous epithelium cycle was determined by cell position, nuclear morphology, and morphology of acrosome staining with AlexaFlour 568-conjugated lectin PNA. 
$734 \quad$ Fig 3. Cytological analysis of ${\text { Kctd } 19^{d e l / d e l}}^{\text {spermatocytes. }}$

(A \& B) Immunostaining of spread nuclei from prophase spermatocytes collected from adult

(A) and juvenile (B) mice. (C) The percentage of each meiotic prophase stage present is

determined by immunostained spread nuclei samples. (D) Giemsa staining of spread nuclei

of metaphase I spermatocytes. (E) Immunostaining of prophase spermatocytes with antibodies against CENPC and $\alpha$-TUBULIN. Right panels $(1-4)$ show additional Kctd19 $9^{\text {del/del }}$ spermatocytes. Red arrows indicate misaligned chromosomes. $(F)$ The percentage of metaphase I spermatocytes with misaligned chromosomes. (G) Immunostaining of prophase spermatocytes with antibodies against SYCP3 and $\alpha$ TUBULIN. Right panels $(1-4)$ show additional Kctd19del/del spermatocytes. Red and yellow arrows indicate misaligned chromosomes and SYCP3 polycomplexes, respectively. $(\mathrm{H})$ The percentage of metaphase I spermatocytes with SYCP3 polycomplexes.

Fig 4. Transgenic (Tg) rescue of Kctd19 ${ }^{\text {del/del }}$ mice.

(A) Schematic of Tg mouse production. Red and yellow boxes indicate affinity tags and Kctd19

ORF, respectively. Black arrows indicate primers for genotyping. (B) An example of PRC genotyping with two primer sets shown in A. (C \& D) Immunoblotting with antibodies against FLAG (C) and HA (D) for determining expression levels. (E \& F) Immunoprecipitation with antibodies against FLAG $(E)$ and $H A(F)$ and immunoblotting with antibodies against $H A$ and FLAG, respectively. (G) Schematics of fertility determination of Tg mice. (H \& I) Two-cell embryos obtained from WT females mated with Kctd19del/del males with transgenes. $(\mathrm{J})$ Testis morphology and $(\mathrm{K})$ testis/body weight of WT and Kctd19del/del, $\mathrm{Tg} \# 1$ adult mice at 8 weeks.

756 Testis/body weight: $3.2 \pm 0.1$ [WT]; $4.7 \pm 1.6$ [Kctd19del/del, Tg \#1]. Error bars indicate one 
standard deviation. (L) PAS staining of seminiferous tubules of adult mice. The seminiferous epithelium cycle was determined by germ cell position and nuclear morphology. (M) Immunostaining of seminiferous tubules of adult mice. The seminiferous epithelium cycle was determined by cell position, nuclear morphology, and morphology of the acrosome stained with AlexaFlour 568-conjugated lectin PNA.

\section{Fig 5. IP-MS analysis with anti-KCTD19 antibody}

(A \& B) Silver staining of IP eluting samples with rabbit $p A b(A)$ and rat $m A b \# 1$ (B). Two juvenile WT mice and two adult Kctd19del/del mice were used for each experiment. (C) The list of identified proteins by MS analysis. The quantitative value was calculated using Scaffold software (D) Immunoprecipitation with an anti-HDAC1 antibody and immunoblotting with an anti-HDAC1 antibody. For input sample, $50 \mu \mathrm{g}$ of testis lysate was used. (E) Immunoblotting with an anti-HDAC1 antibody. GPADH was used as a loading control. (F) Immunostaining with an anti-HDAC1 antibody. The seminiferous epithelium cycle was determined by cell position, nuclear morphology, and morphology of the acrosome stained with AlexaFlour 568-conjugated lectin PNA.

Fig 6. Chimeric analysis of Zfp541 KO spermatocytes

(A) RT-PCR using multi-tissue cDNA. Actb was used as a loading control. (B) RT-PCR using postnatal testis cDNA. Actb was used as a loading control. (C) Gene map of Zfp541. Black and white boxes indicate coding and non-coding regions, respectively. Black arrows and arrowheads indicate primers for genotyping and gRNAs for genome editing, respectively. (D) of chimeric mice production. ESC-derived cells were labeled with GFP fluorescence. (F) PAS 
staining of seminiferous tubules of chimeric mice. ES cell-derived Zfp541-KO spermatocytes were identified by immunohistochemistry against GFP. (G) TUNEL staining of seminiferous tubules of chimeric mice counterstained with hematoxylin. ES cell-derived Zfp541-KO spermatocytes were identified by immunohistochemistry against GFP. (H \& I) Immunostaining of seminiferous tubules of chimeric mice with antibodies against KCTD19 $(\mathrm{H})$ and HDAC1 (I).

786 ES cell-derived Zfp541-KO spermatocytes were identified by GFP immunostaining. (J)

787 Immunostaining of spread nuclei of prophase spermatocytes collected from XY->XX chimeric mice. Red and yellow boxes were magnified in the right panels. $(K)$ The percentage of cells in various meiotic prophase stages counted with immunostained spread nuclei samples. 


\section{Supporting information}

Fig S1. Sequence comparison of KCTD19 in various mammals.

794 Prortein sequence comparison of KCTD19 in cattle (NP_001098862.1), pig (XP_003126977.2), dog (XP_022275030.1), fox (XP_025867456.1), cat (XP_023101865.1), bat (XP_027998908.1), human (NP_001094385.1), chimpanzee (XP_523391.2), rhesus monkey (XP_014981866.1), mouse (NP_808459.1), rat (NP_001292128.1), and golden hamster (XP_021086458.1).

799

Fig S2. Production of Kctd19- $\mathrm{POZ}$ mice and fertility analysis.

801

(A) Gene map of Kctd19. Black and white boxes indicate coding and non-coding regions, respectively. Black arrows and arrowheads indicate primers for genotyping and gRNAs for genome editing, respectively. (B) An example of genotyping PCR with two primer sets shown in S2A. (C) DNA sequencing verify the deletion. (D) RT-PCR using tetis cDNA obtained from WT and $\triangle P O Z / \triangle P O Z$ mice. Actb was used as a loading control. (E) Immunoblotting using tetis lysates obtained from WT, del/del, and $\triangle P O Z / \triangle P O Z$ mice. (F) PAS staining of seminiferous tubules of adult mice. The seminiferous epithelium cycle was determined by germ cell position and nuclear morophology.

Fig S3. Sequence comparison of ZFP541 in various mammals.

811 Prortein sequence comparison of ZFP541 or ZNF541 proteins from various mammals: cattle

812 (XP_015313711.2), pig (XP_020950303.1), dog (XP_005616437.1), fox (XP_025869832.1), 813 cat (XP_023100994.1), bat (XP_008152641.1), human (NP_001264004.1), chimpanzee 814 (XP_016791837.1), rhesus monkey (XP_014979842.2), mouse (NP_001092747.1), rat 815 (NP_001100928.2), and golden hamster (XP_021078928.1). 
bioRxiv preprint doi: https://doi.org/10.1101/2021.02.12.430913; this version posted February 12, 2021. The copyright holder for this preprint (which was not certified by peer review) is the author/funder, who has granted bioRxiv a license to display the preprint in perpetuity. It is made available under aCC-BY 4.0 International license.

816

817 Fig S4. Production of $\mathbf{X Y}->\mathbf{X X}$ chimeric mice and their feature.

818 (A) Schematic of $X Y->X X$ chimeric mice production. $X X$ prospermatogonia are eliminated

819 around PND2. (B) Testis section from chimeric mice. ES cell-derived cells were labeled with

820 GFP fluorescence. Astarisk indicates depleted tubules. 


\section{Figure1}

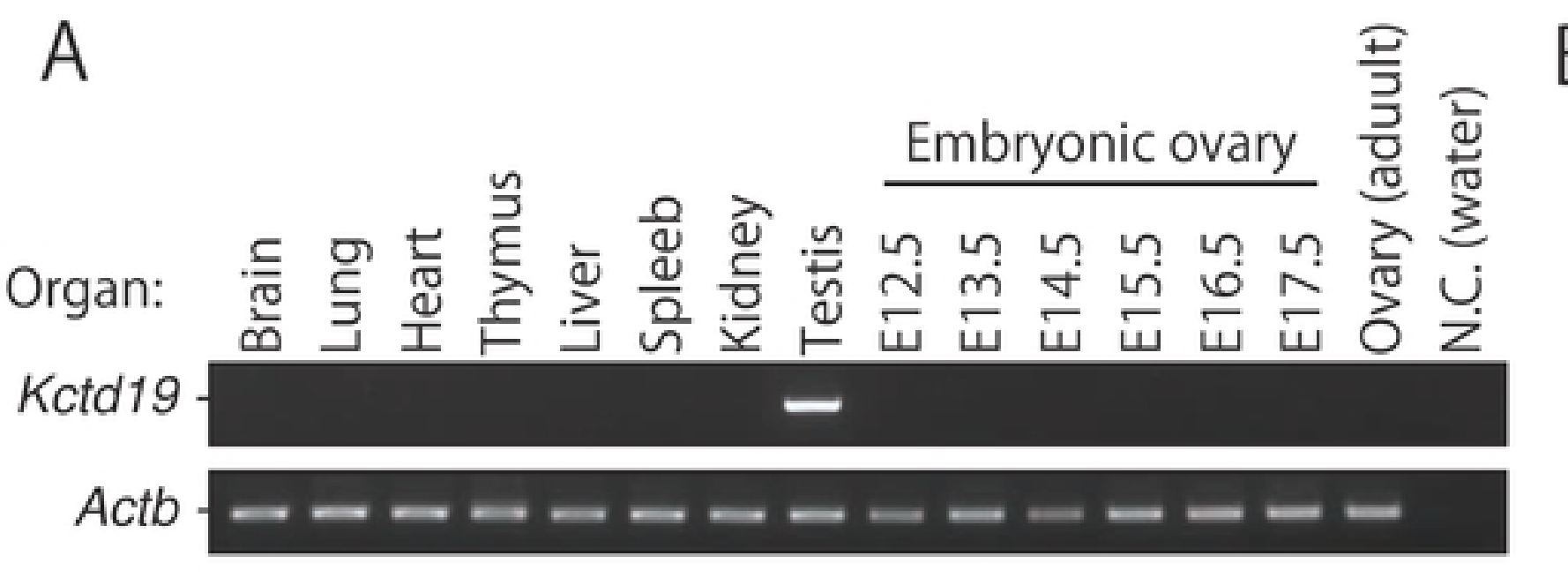

C

Antigen for Rabbit pAb

\section{BTB}

747_ 765

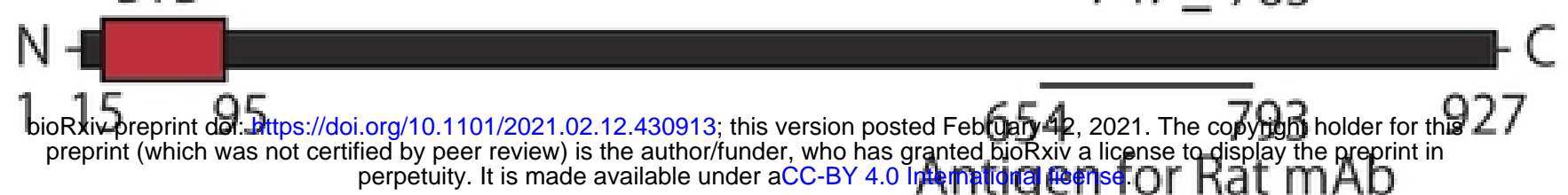

$\mathrm{E}$

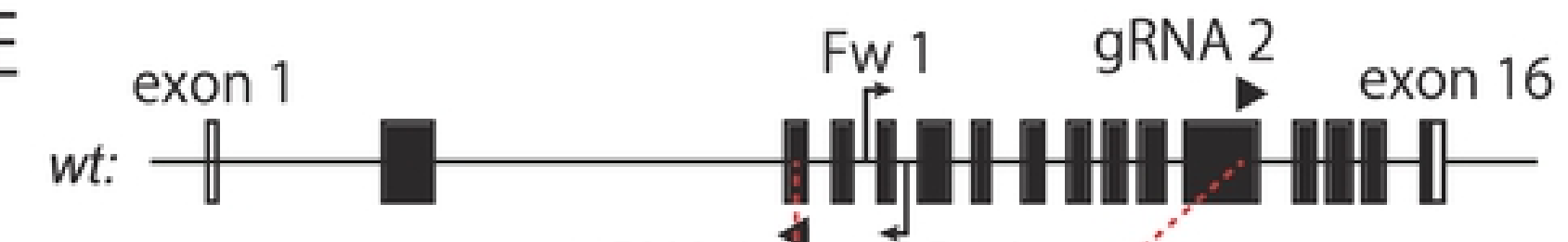

D

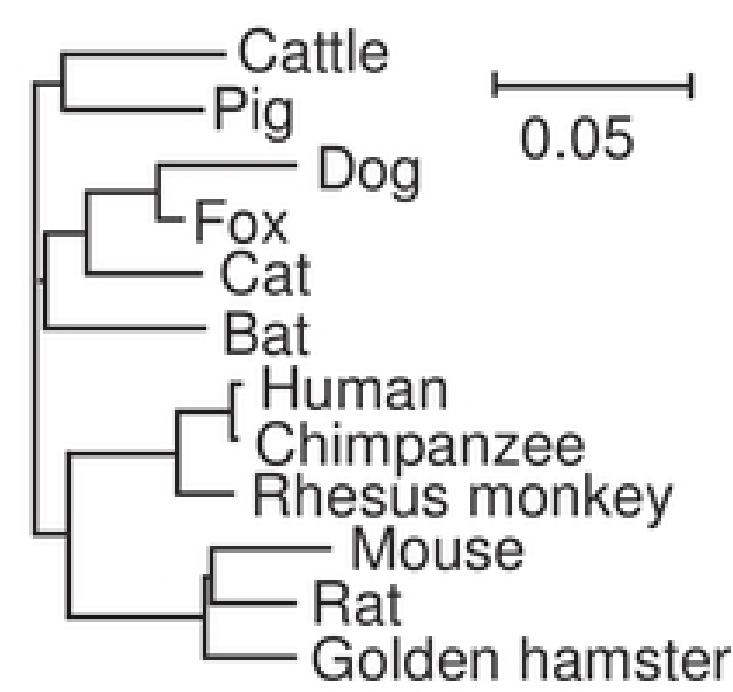

del:

gRNA 1

Fw 2

$-9620 \mathrm{bp}$

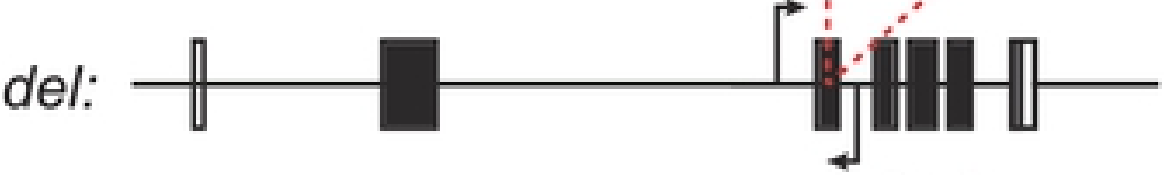

Rv 2

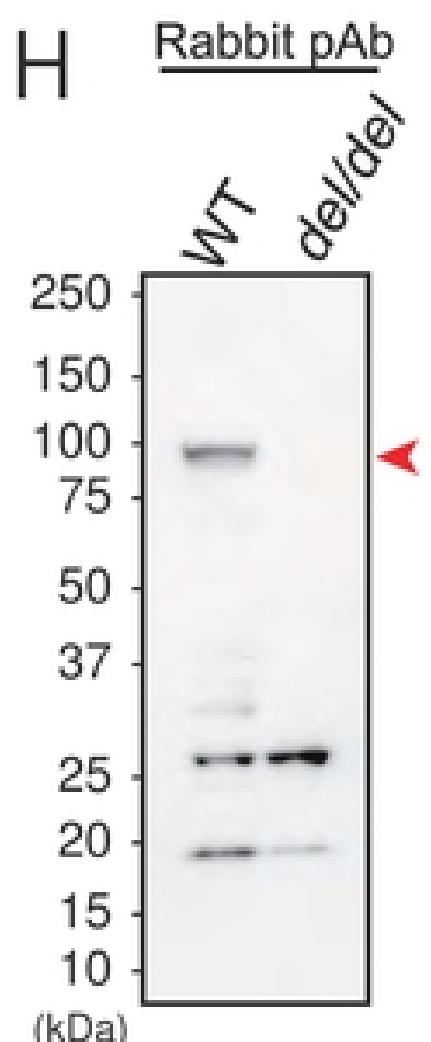

GAPDH
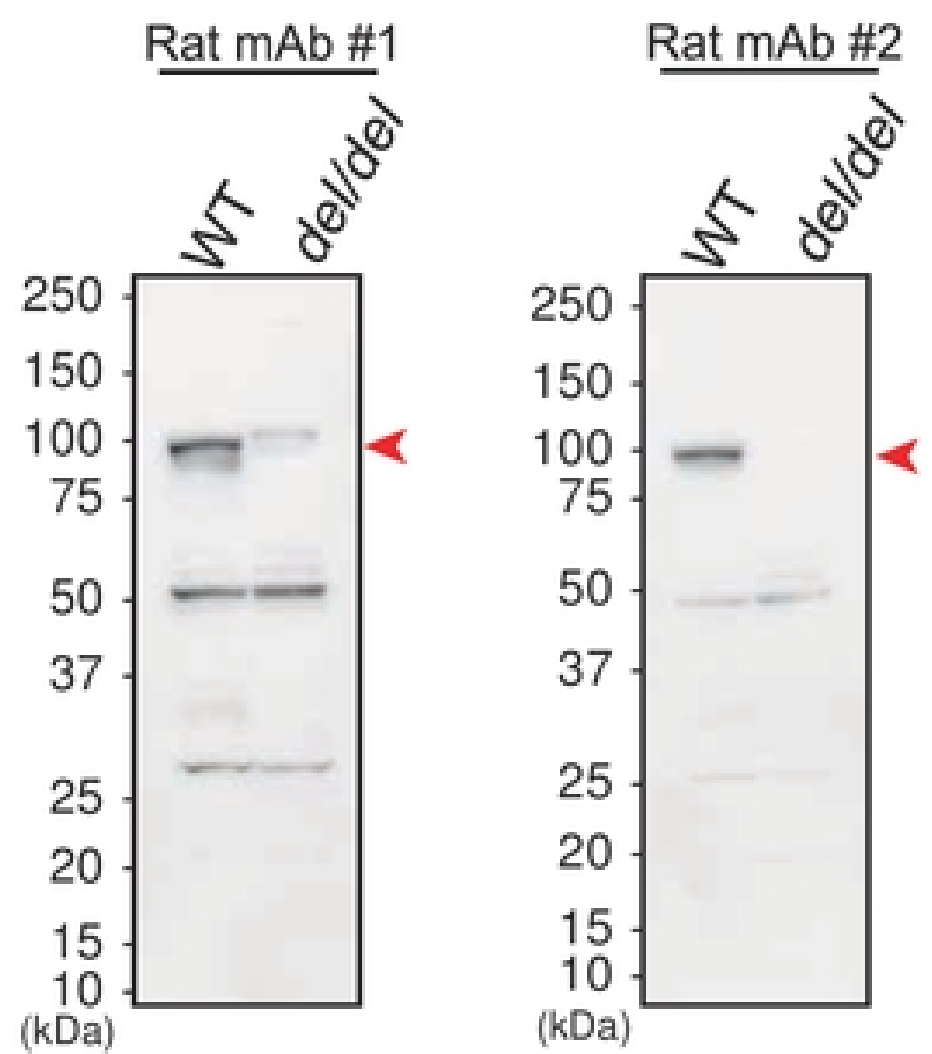

Expected size : 107 kDa
Tesits

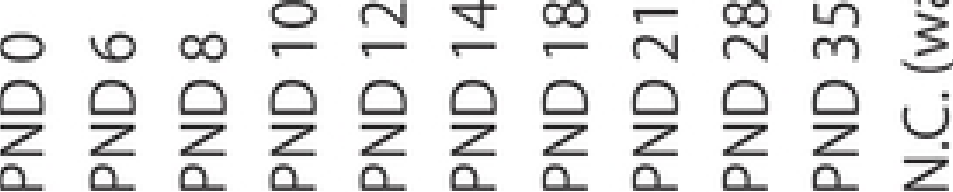

\section{-}

- 


\section{Figure2}
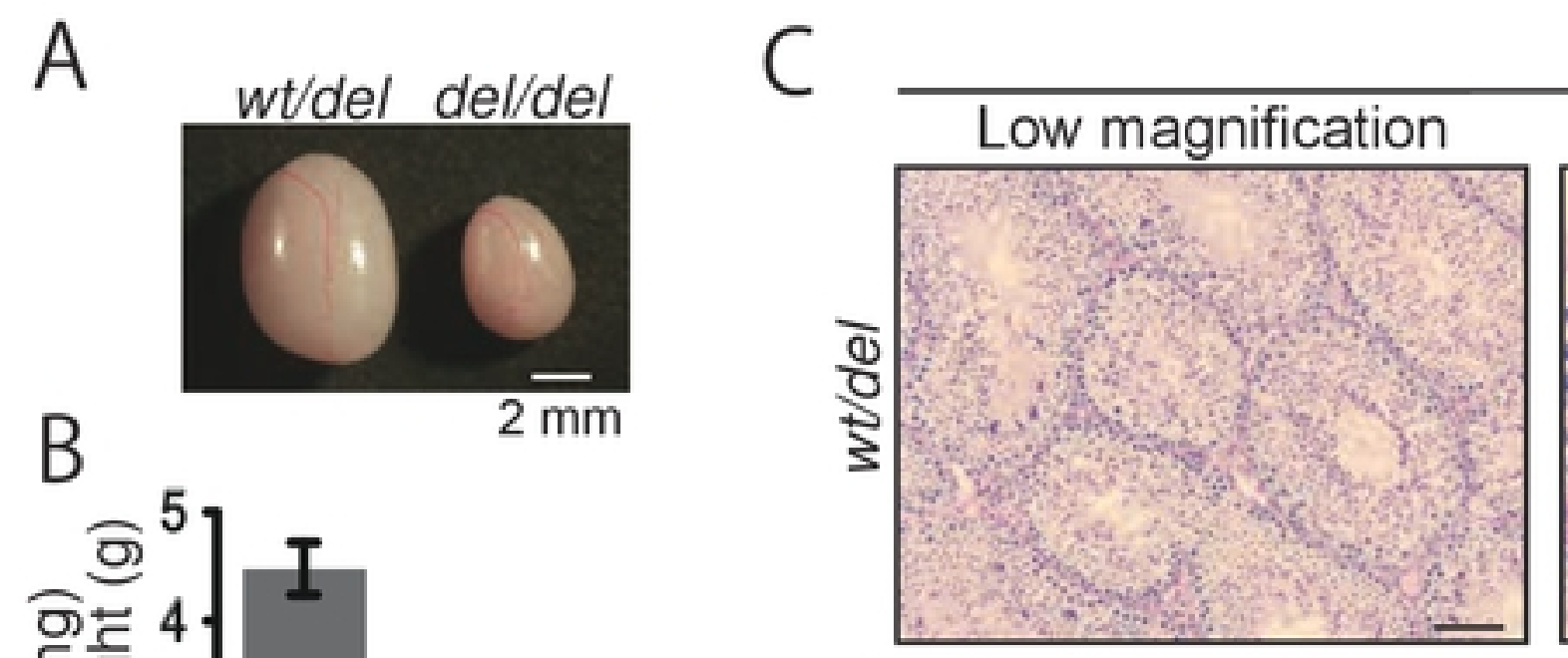

HePAS (Testis)
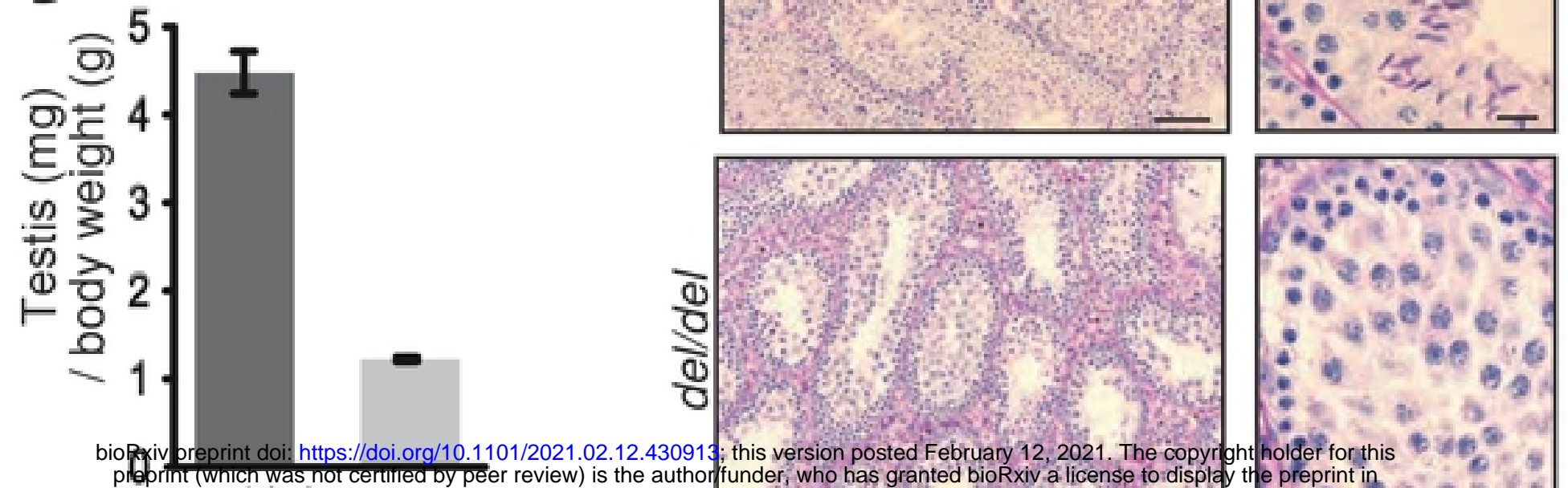

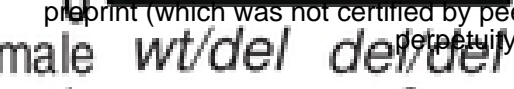

\# of male 4
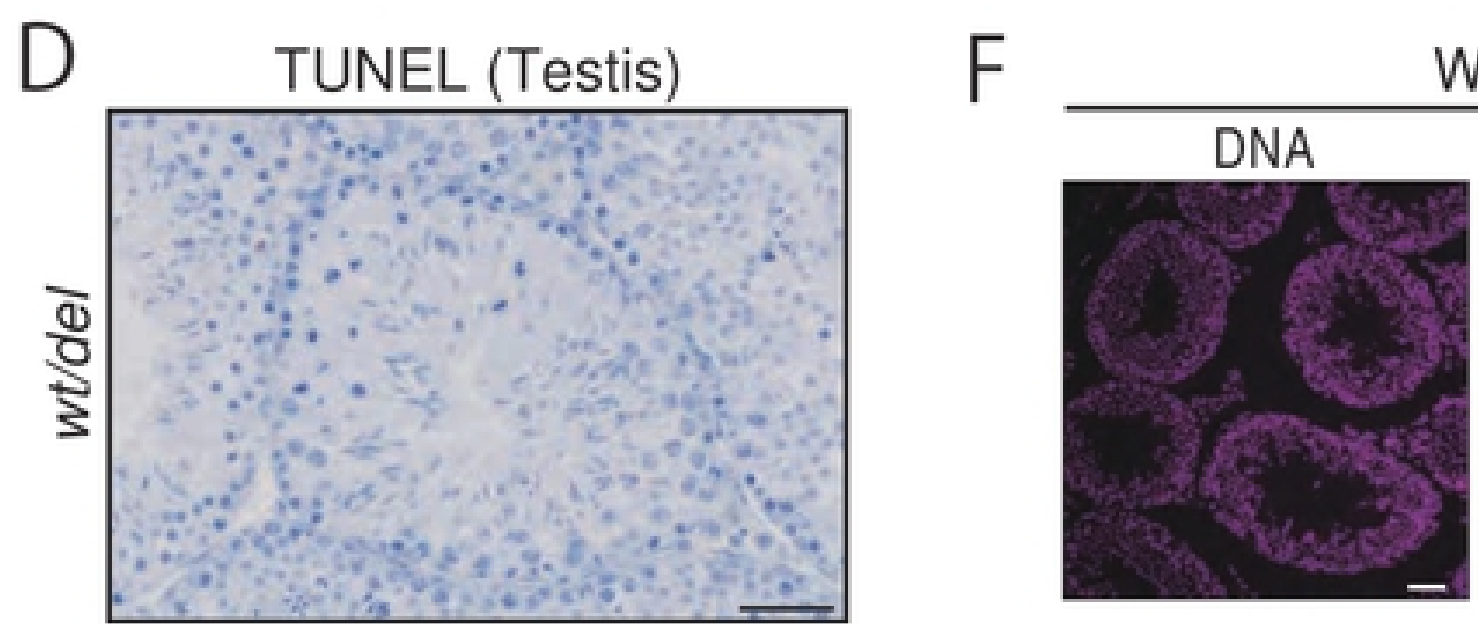

$100 \mu \mathrm{m}$
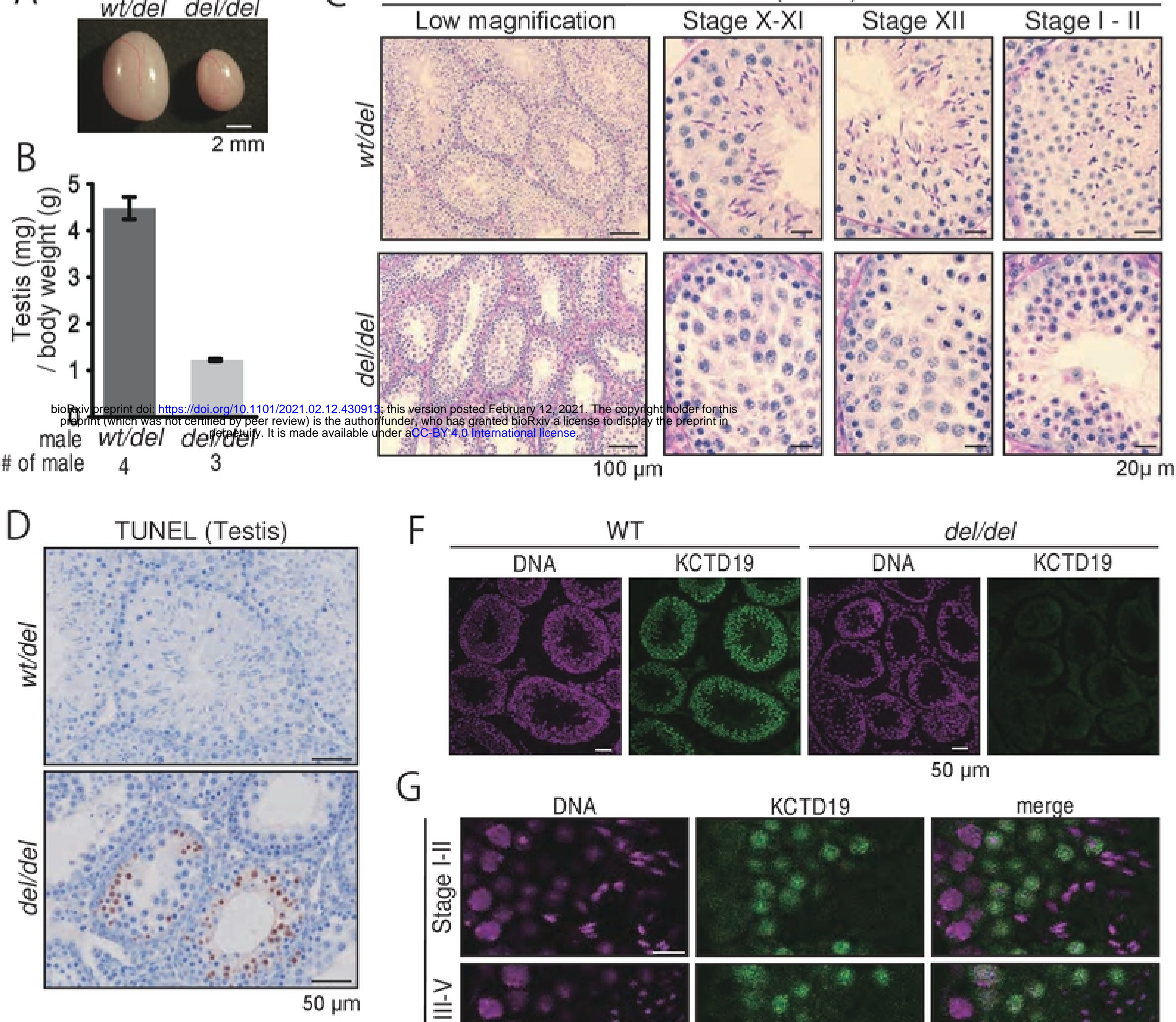

$G$

E HePAS (Cauda epididymus)
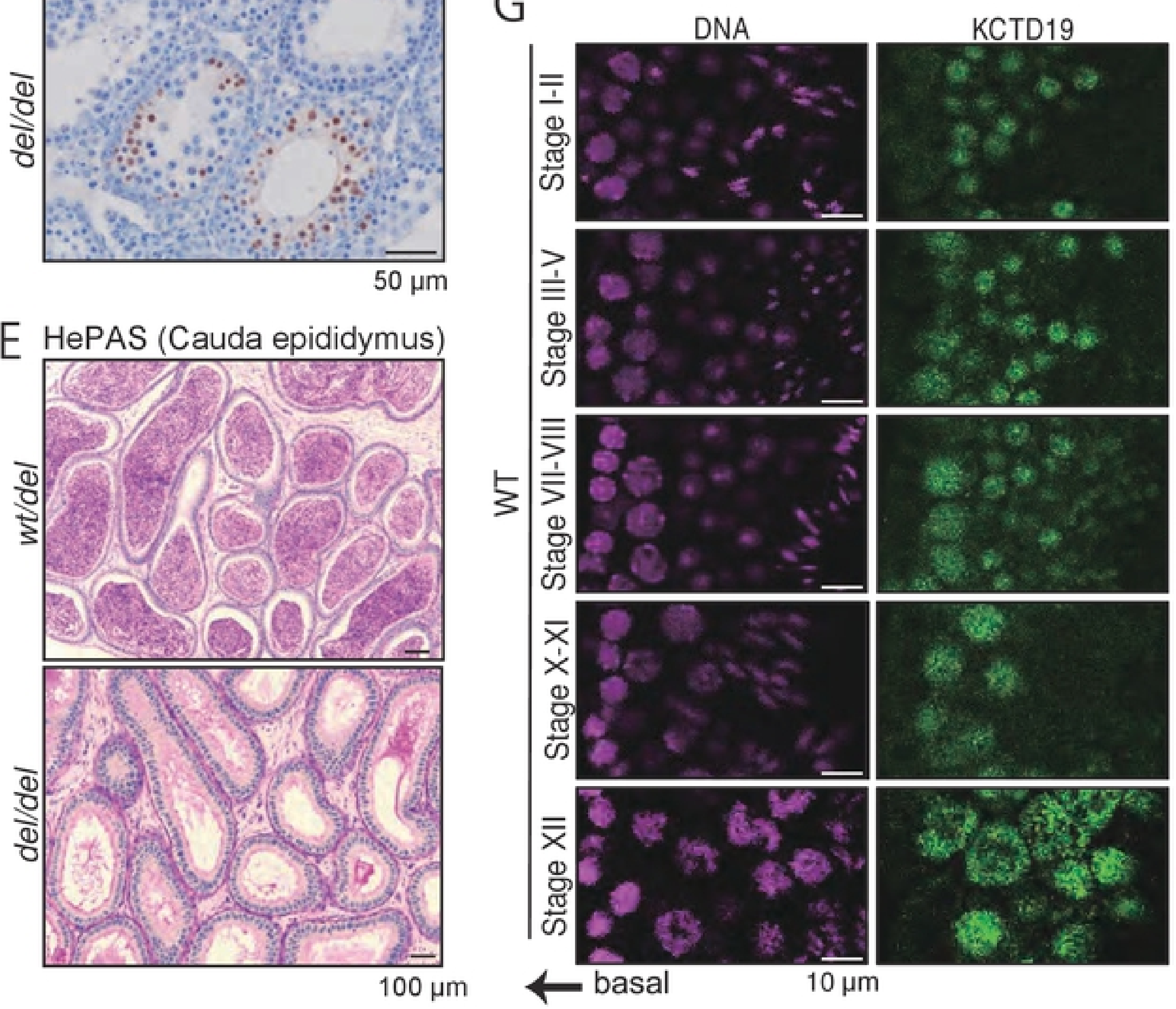

$50 \mu \mathrm{m}$ 


\section{Figure3}

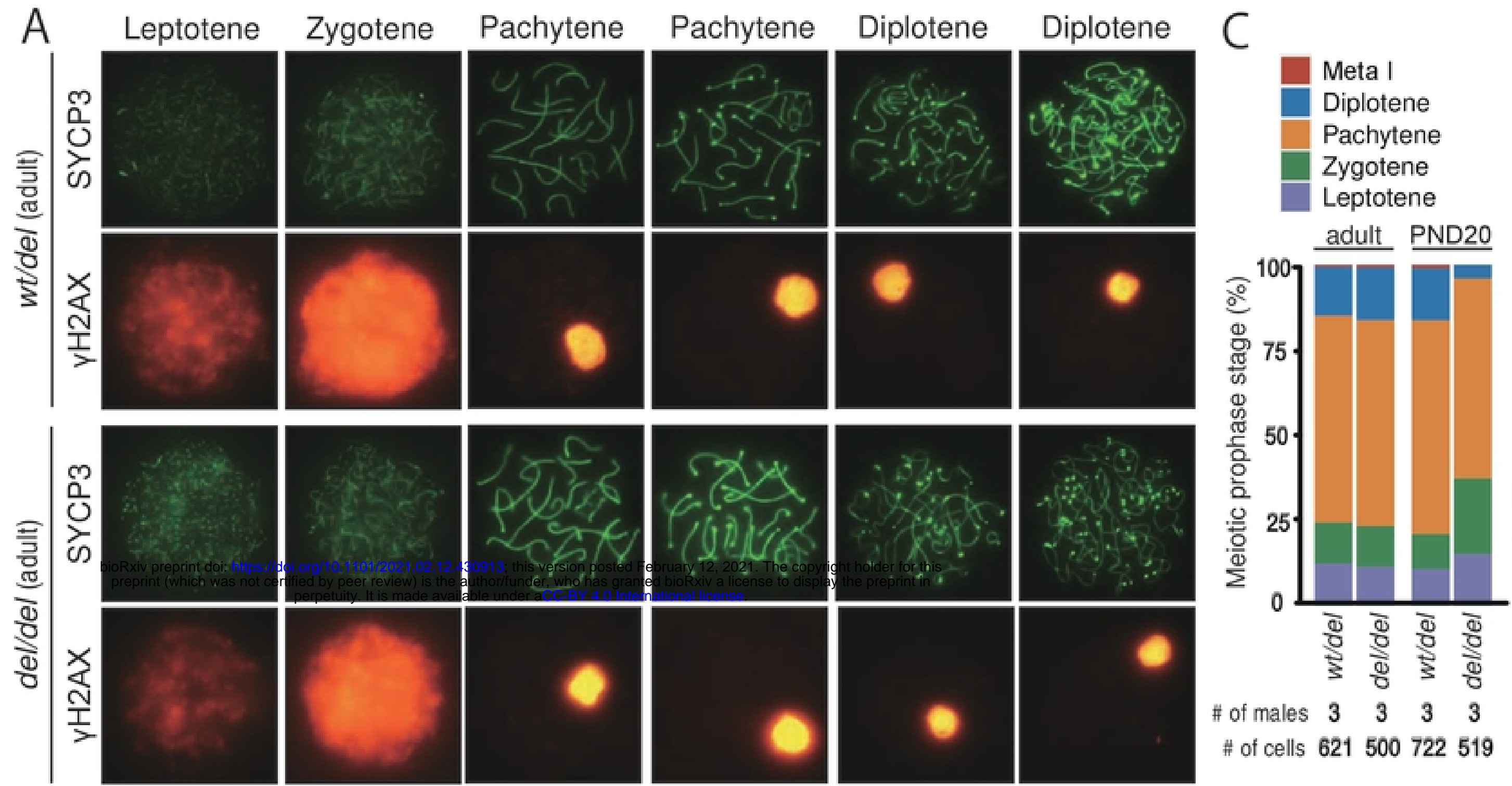

B
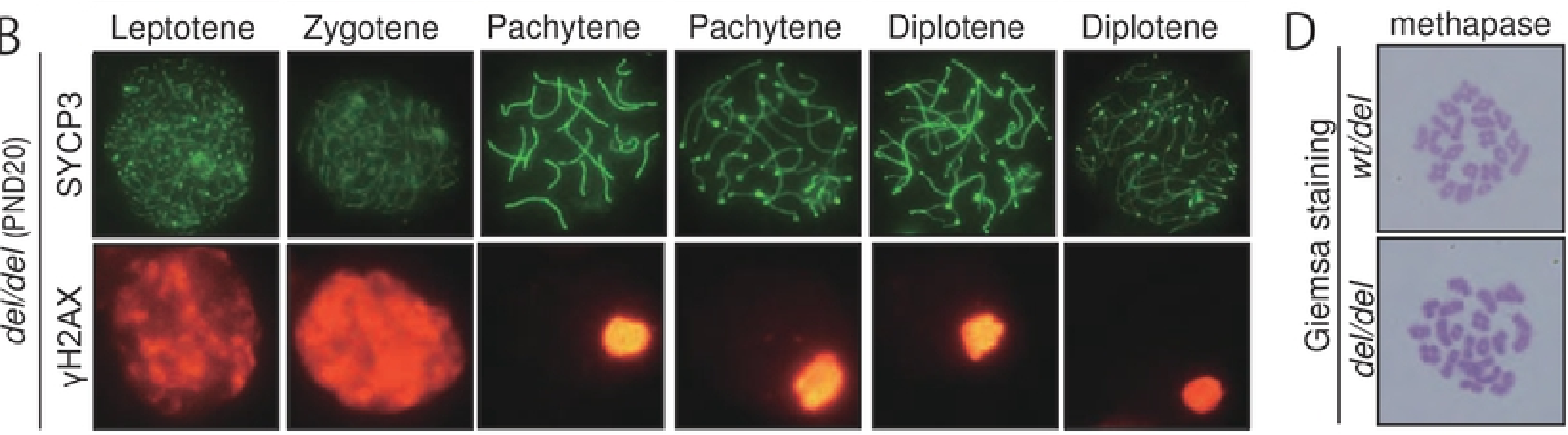

E

$\frac{8}{8}$
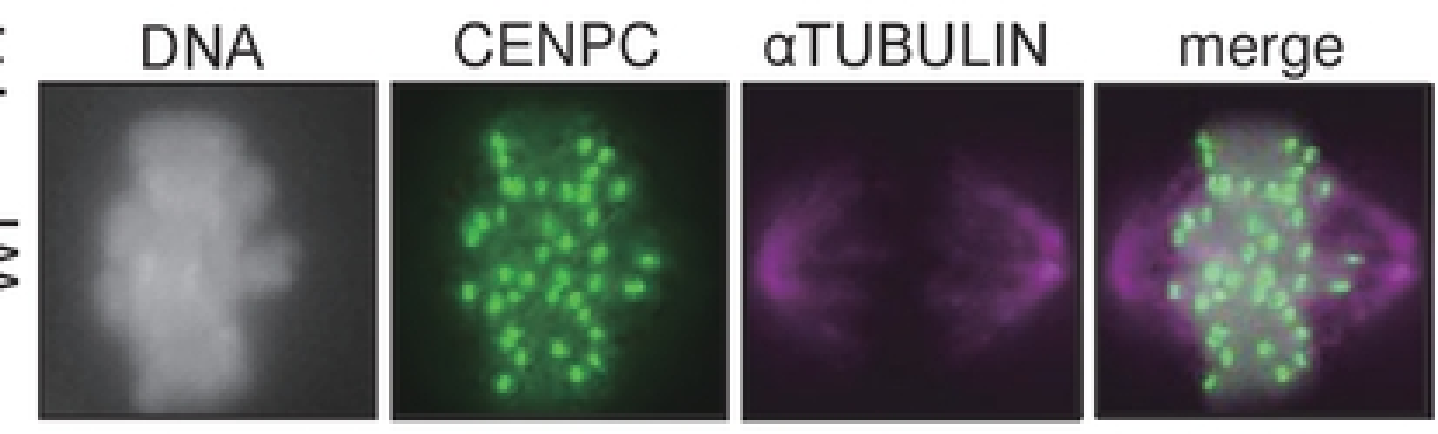

ఫ్ర

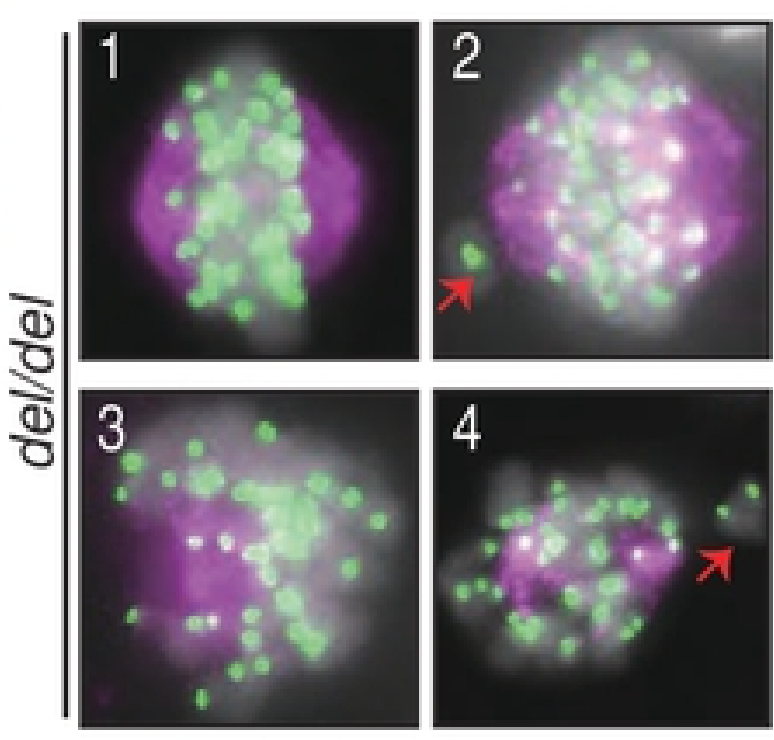

5
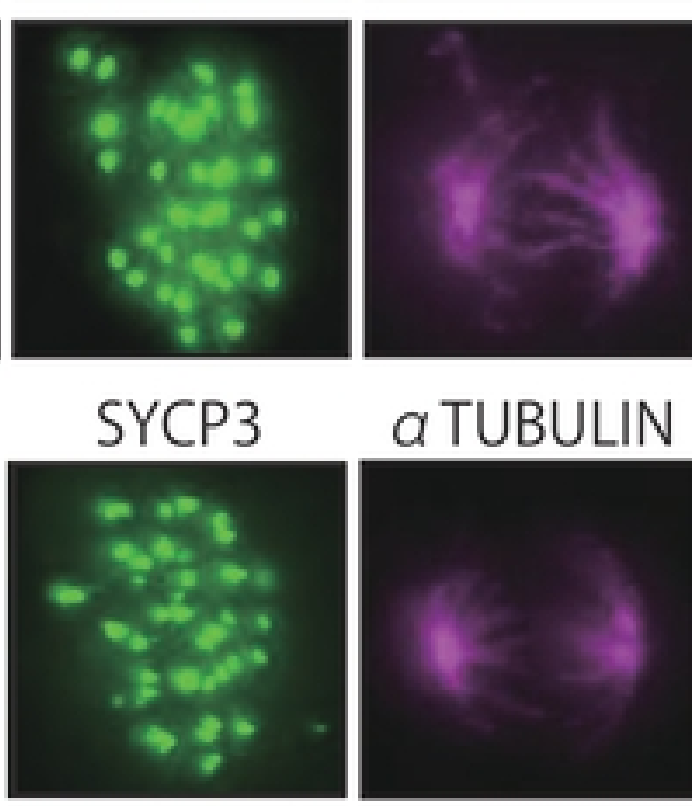

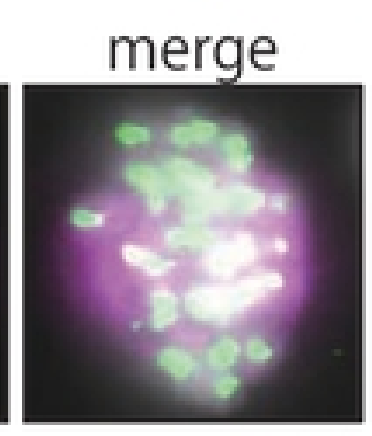

$y$.

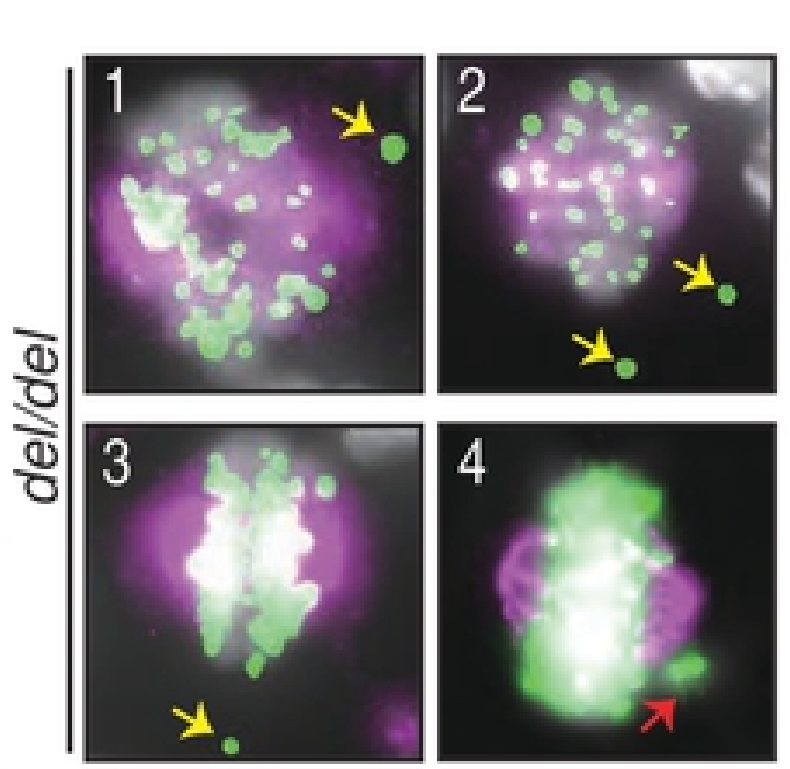

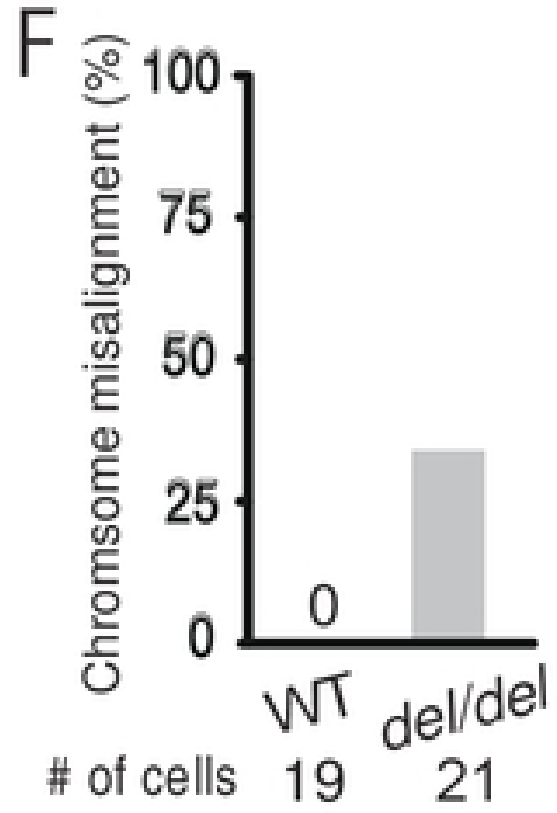

$\mathrm{H}$

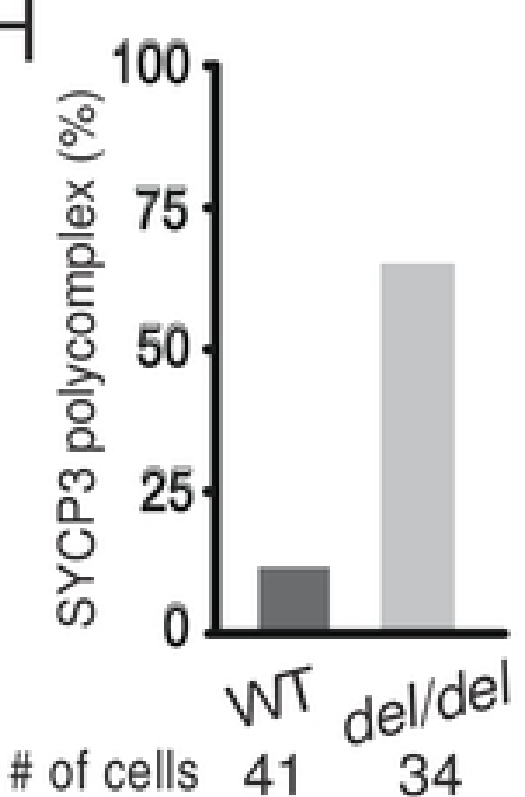




\section{Figure4}
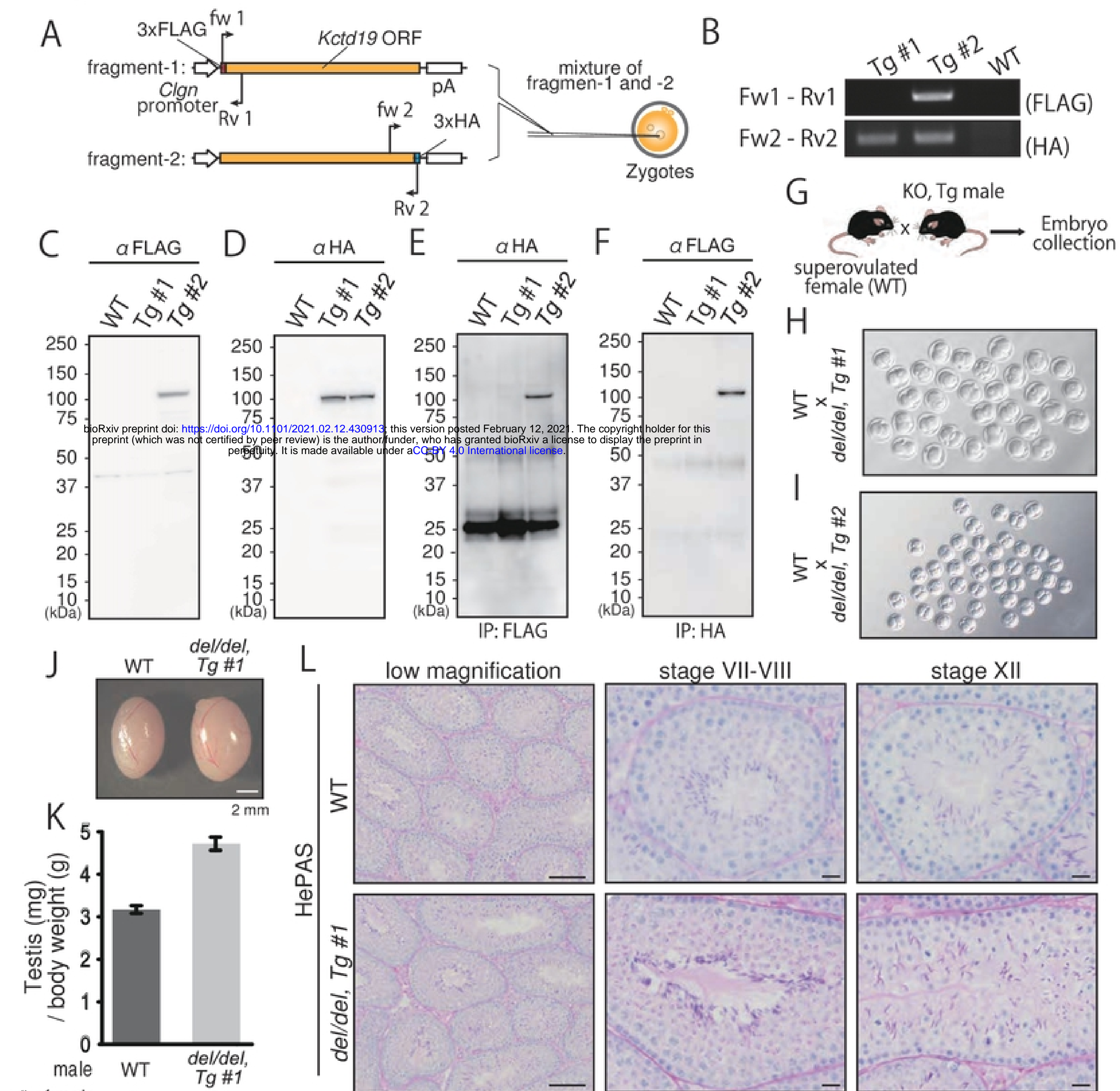

\# of male 4
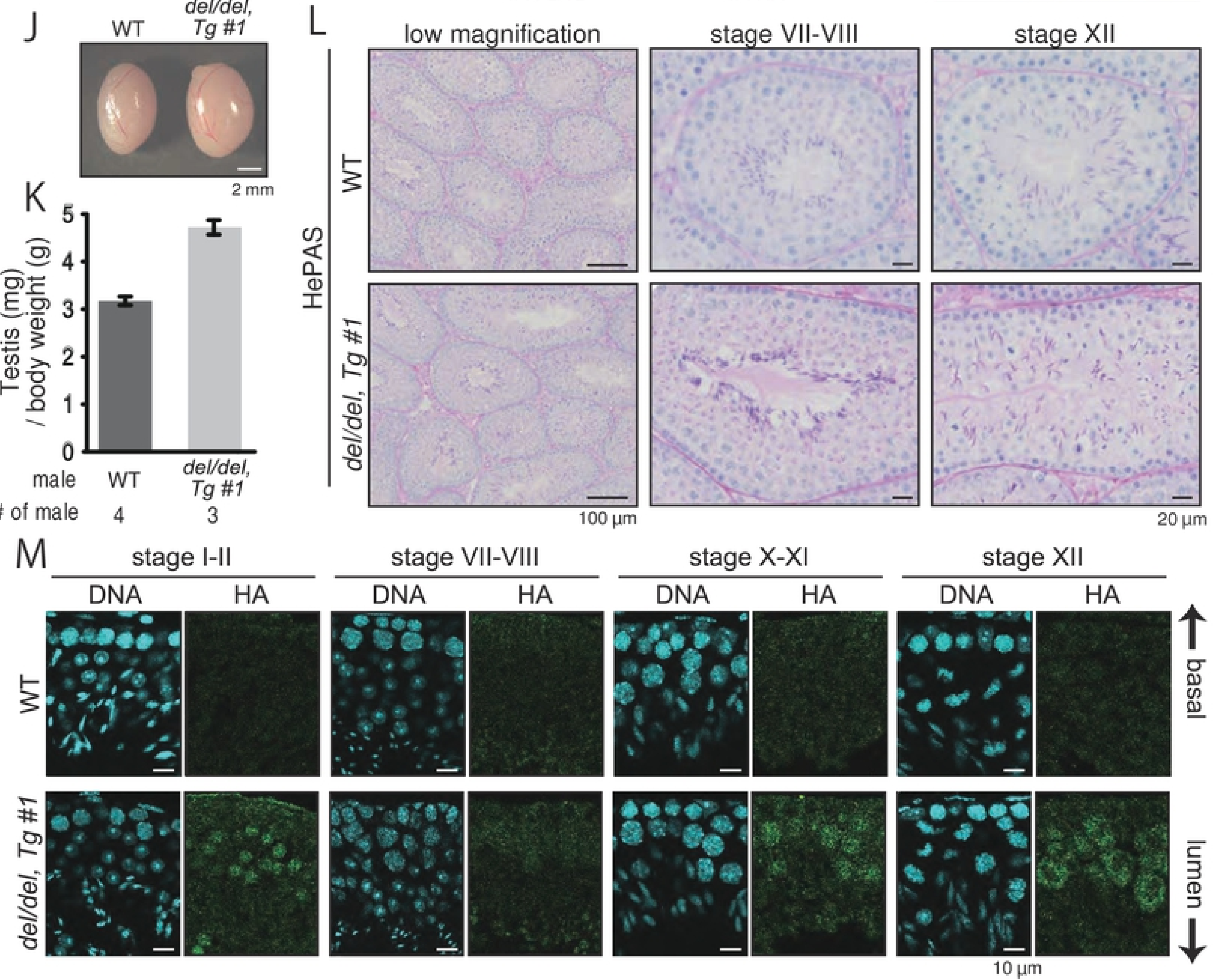


\section{Figure5}

A

IP: Rabbit pAb $\quad$ B IP: Rat pAb\#1 C

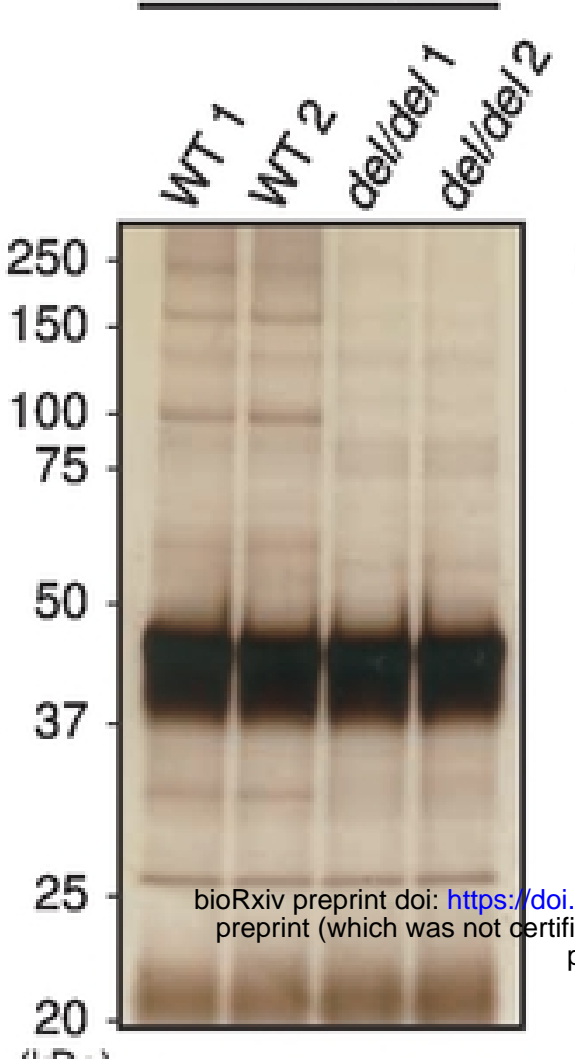

(kDa)

D

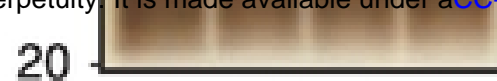

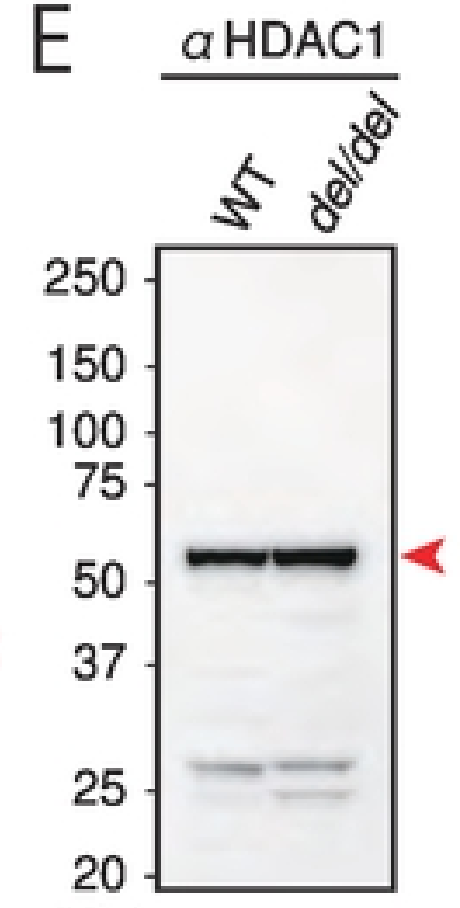

(kDa)

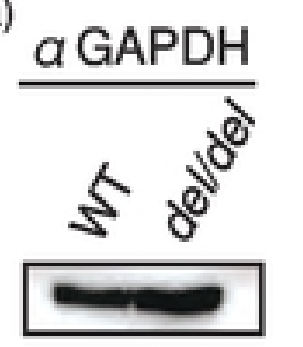

Quantitative Value (Normalized Total Spectra)

WT (PND21)

Protein MW Accession Rabbit pAb Rat mAb \#1 Rabbit $\mathrm{pAb}$ Rat mab \#1 Number $\quad(\mathrm{N}=1) \quad(\mathrm{N}=2) \quad(\mathrm{N}=1) \quad(\mathrm{N}=2) \quad(\mathrm{N}=1) \quad(\mathrm{N}=2) \quad(\mathrm{N}=1) \quad(\mathrm{N}=2)$

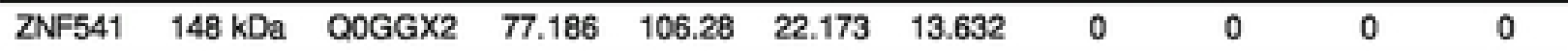

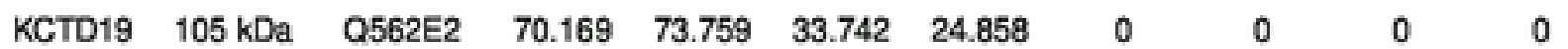

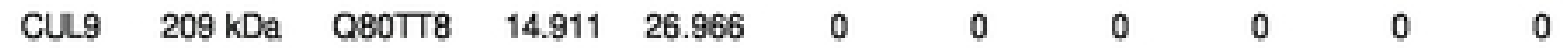

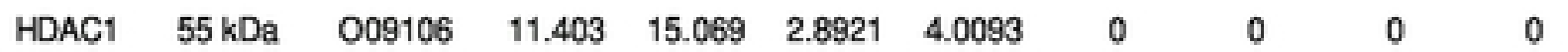

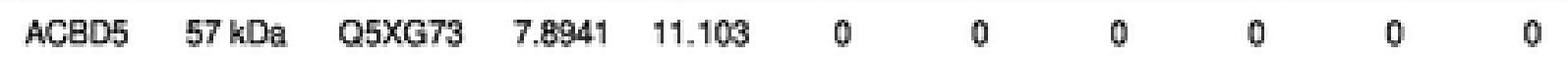

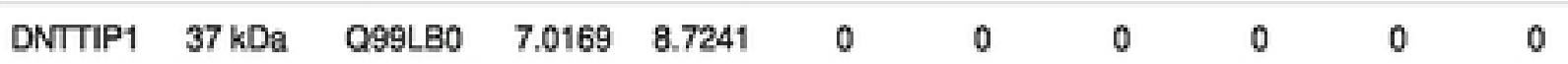

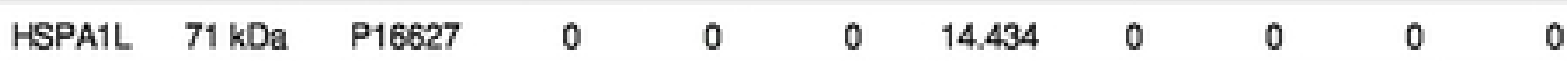

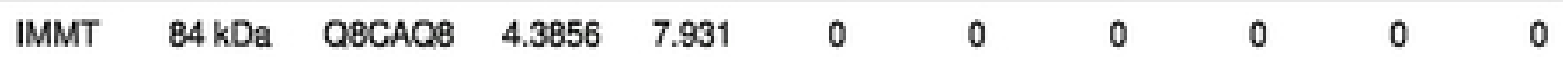

$\begin{array}{llllllllll}\text { PEX5 } & 71 \mathrm{kDa} & 009012 & 6.1398 & 5.5517 & 0 & 0 & 0 & 0 & 0\end{array}$

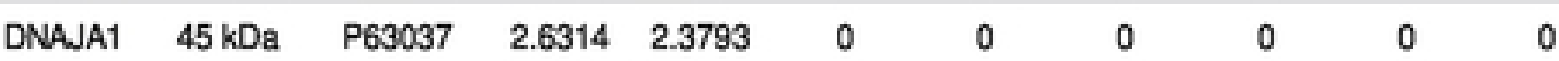

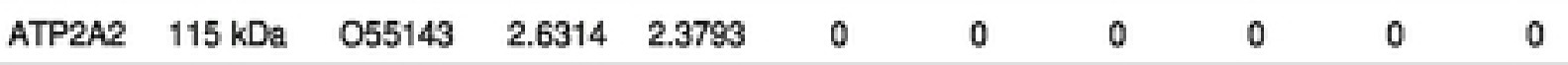

$\begin{array}{llllllll}\text { s.on posted February 12, 2021. The copyright holder for this } & 2.3793 & 0 & 0 & 0 & 0 & 0 & 0\end{array}$

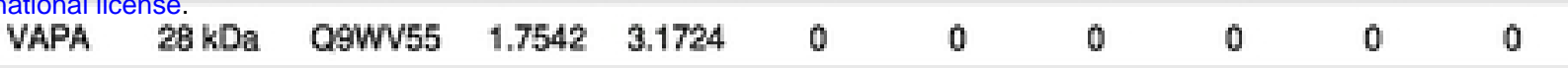

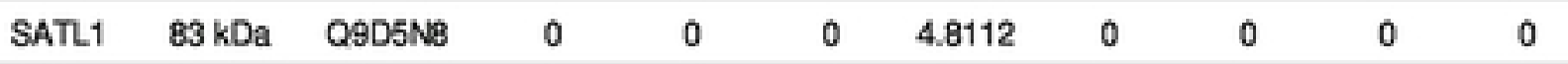

$\begin{array}{llllllllll}\text { MAVS } & 53 \mathrm{kDa} & \text { QBVCFO } & 2.6314 & 1.5862 & 0 & 0 & 0 & 0 & 0\end{array}$

$\begin{array}{llllllllll}\text { RPL18 } & 22 \mathrm{kDa} & \text { P35980 } & 2.6314 & 1.5862 & 0 & 0 & 0 & 0 & 0\end{array}$

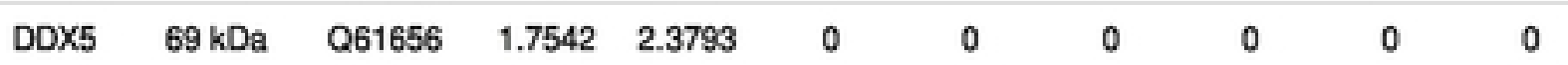

$\begin{array}{llllllllll}\text { SETX } & 298 \mathrm{kDa} & \text { A2AKX3 } & 1.7542 & 1.5862 & 0 & 0 & 0 & 0 & 0\end{array}$

$\begin{array}{lllllllllll}\text { PCBP1 } & 37 \mathrm{kDa} & \mathrm{P} 60335 & 1.7542 & 1.5862 & 0 & 0 & 0 & 0 & 0 & 0\end{array}$

$\begin{array}{llllllllll}\text { RPL3 } & 46 \mathrm{kDa} & \mathrm{P} 27659 & 1.7542 & 1.5862 & 0 & 0 & 0 & 0 & 0\end{array}$

F StageVII-VIII $\quad$ StageX-XI $\quad$ StageXII

DNA HDAC1 $\quad$ DNA HDAC1 $\quad$ DNA HDAC1
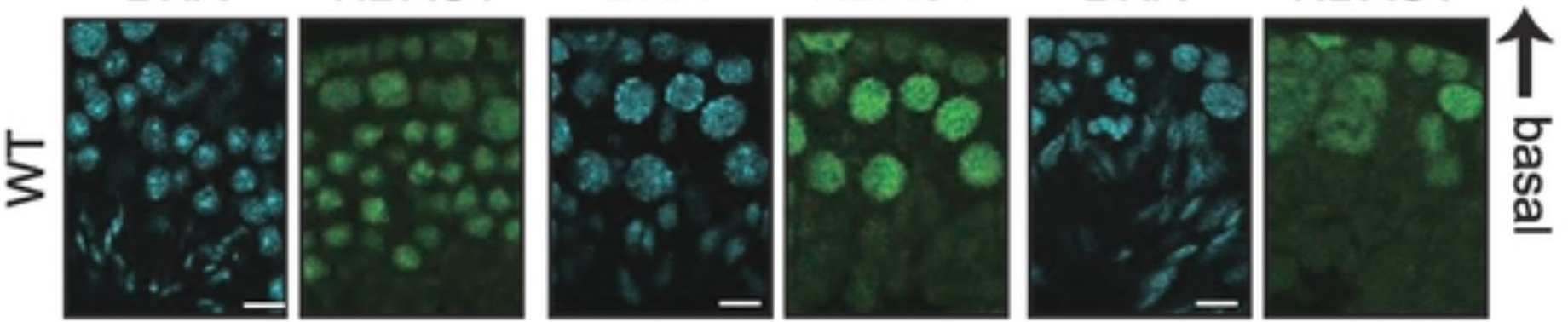

DNA

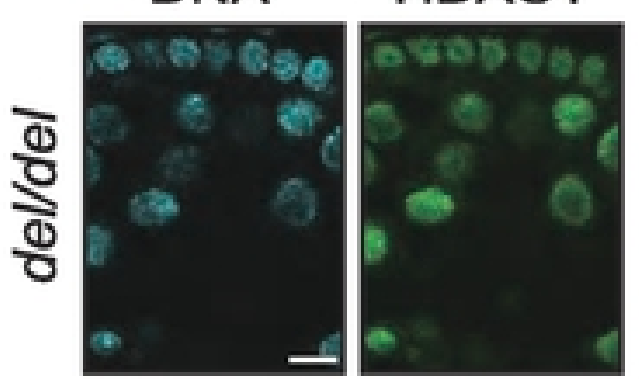

HDAC1

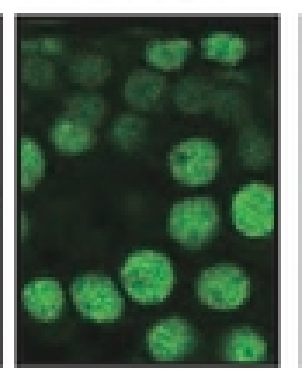

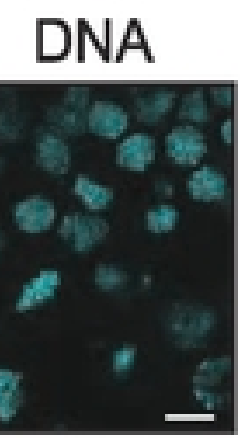

HDAC1
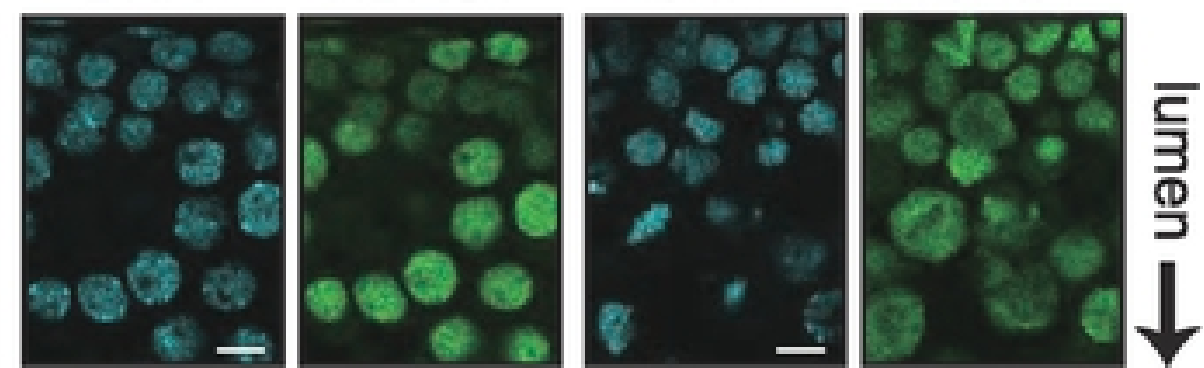

$10 \mu \mathrm{m}$ 


\section{Figure6}
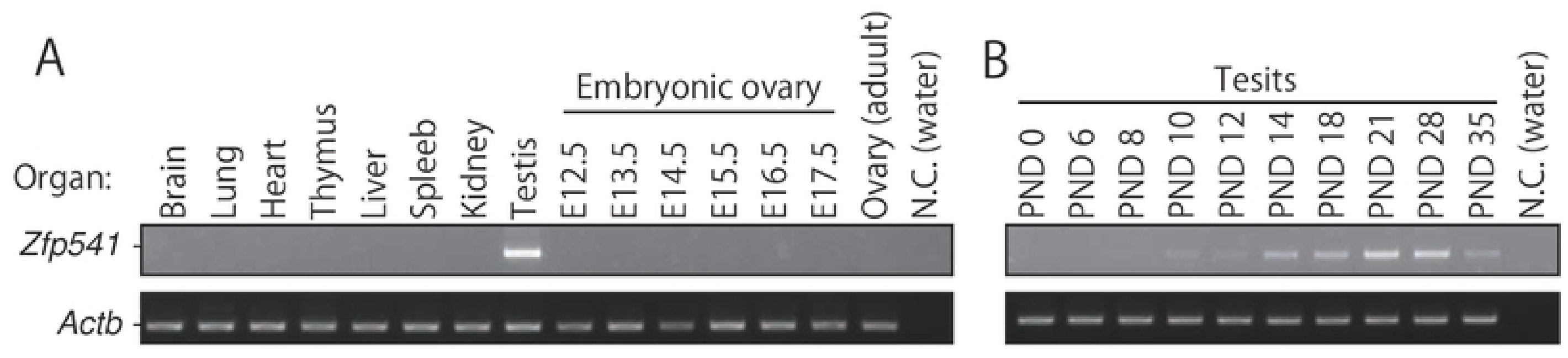
C

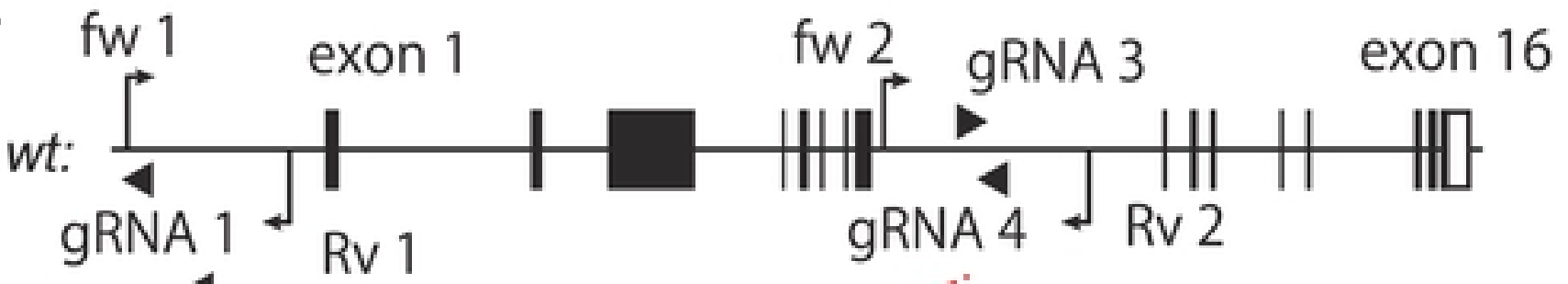
bioRxiv prephint doi: https://doi.org/10.1101/2021.02.12.430913; this version" pósted Febbruary 12, 2021. The copyright holder for this

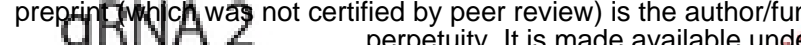
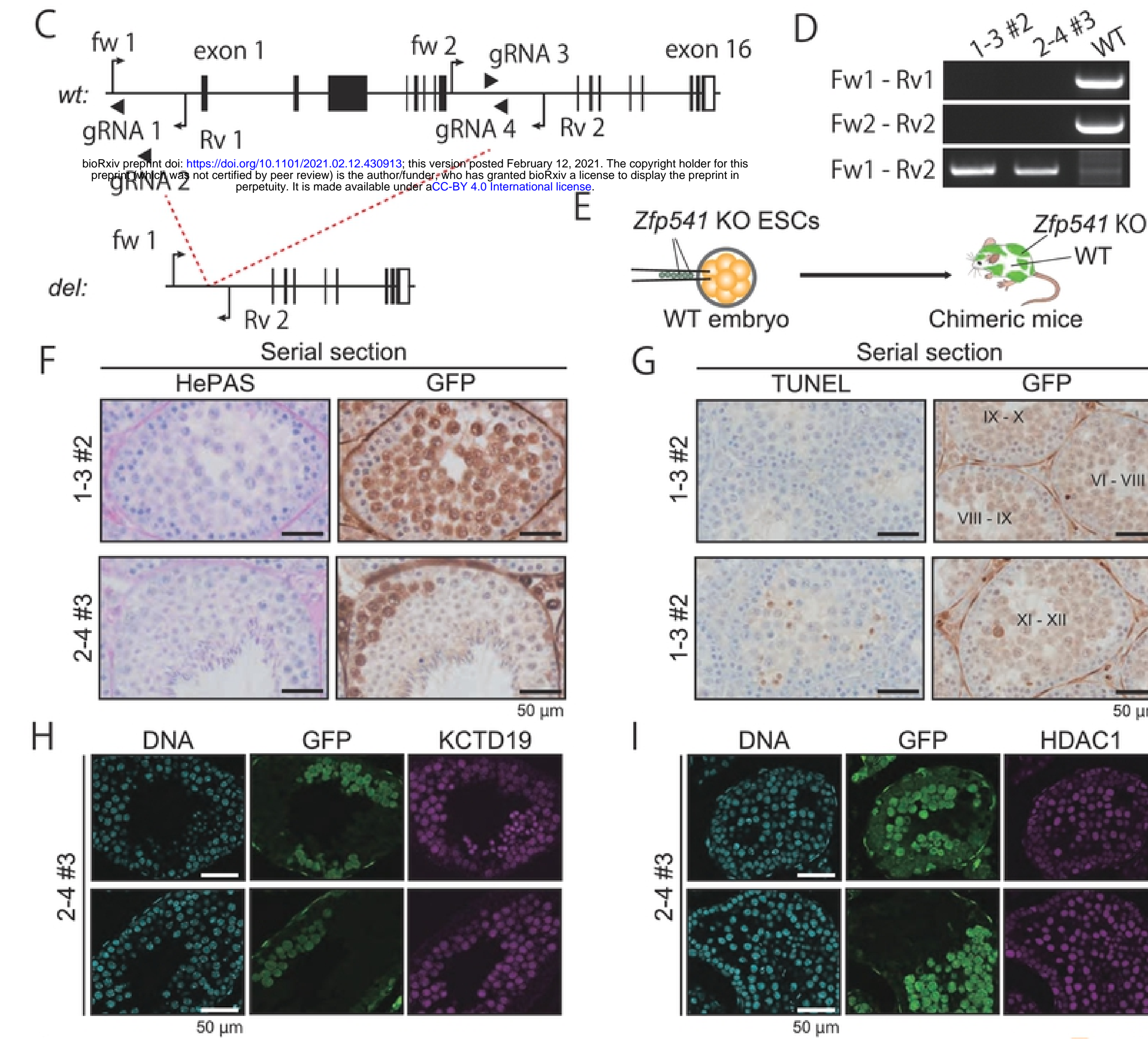

Chimeric mice
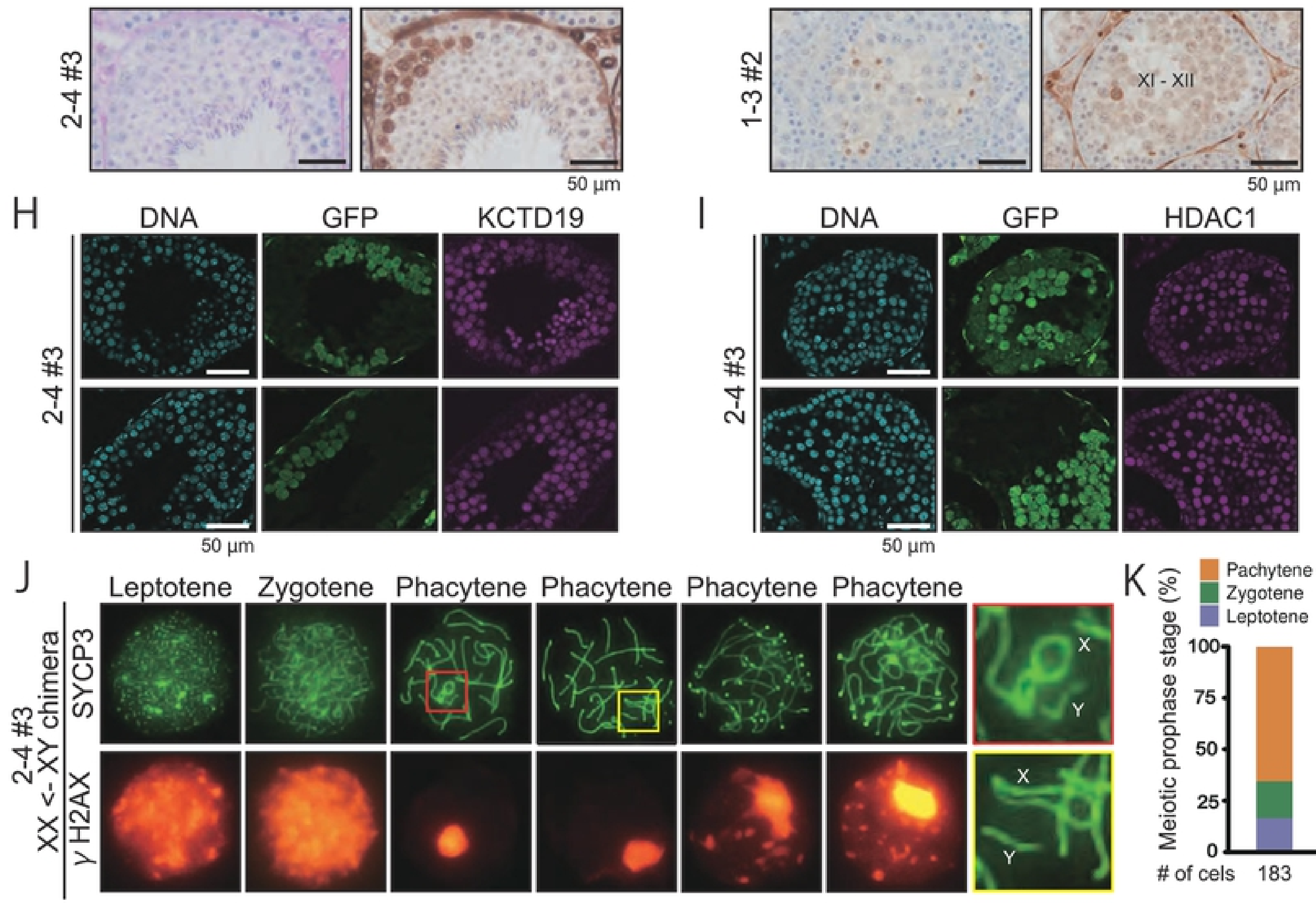

$\mathrm{K} \curvearrowright$ Pachytene

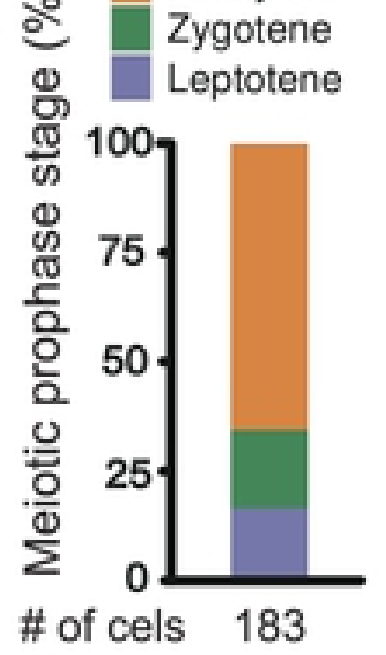

